\title{
Engineering of E. coli inherent fatty acid biosynthesis capacity to increase octanoic acid production
}

Zaigao $\operatorname{Tan}^{1 \dagger}{ }^{\mathbb{D}}$, Jong Moon Yoon ${ }^{1 \dagger}$, Anupam Chowdhury², Kaitlin Burdick${ }^{1}$, Laura R. Jarboe ${ }^{1}$, Costas D. Maranas $^{2}$ and Jacqueline V. Shanks ${ }^{1 *}$

\begin{abstract}
Background: As a versatile platform chemical, construction of microbial catalysts for free octanoic acid production from biorenewable feedstocks is a promising alternative to existing petroleum-based methods. However, the bioproduction strategy has been restricted by the low capacity of E. coli inherent fatty acid biosynthesis. In this study, a combination of integrated computational and experimental approach was performed to manipulate the E. coli existing metabolic network, with the objective of improving bio-octanoic acid production.

Results: First, a customized OptForce methodology was run to predict a set of four genetic interventions required for production of octanoic acid at $90 \%$ of the theoretical yield. Subsequently, all the ten candidate proteins associated with the predicted interventions were regulated individually, as well as in contrast to the combination of interventions as suggested by the OptForce strategy. Among these enzymes, increased production of 3-hydroxy-acyl-ACP dehydratase (FabZ) resulted in the highest increase $(+45 \%)$ in octanoic acid titer. But importantly, the combinatorial application of FabZ with the other interventions as suggested by OptForce further improved octanoic acid production, resulting in a high octanoic acid-producing E. coli strain +fabZ $\triangle$ fadE $\triangle$ fumAC $\triangle a c k A$ (TE10) (+61\%). Optimization of TE10 expression, medium $\mathrm{pH}$, and $\mathrm{C}: \mathrm{N}$ ratio resulted in the identified strain producing $500 \mathrm{mg} / \mathrm{L}$ of $\mathrm{C} 8$ and $805 \mathrm{mg} / \mathrm{L}$ of total FAs, an 82 and 155\% increase relative to wild-type MG1655 (TE10) in shake flasks. The best engineered strain produced with high selectivity (> 70\%) and extracellularly (>90\%) up to $1 \mathrm{~g} / \mathrm{L}$ free octanoic acid in minimal medium fed-batch culture.
\end{abstract}

Conclusions: This work demonstrates the effectiveness of integration of computational strain design and experimental characterization as a starting point in rewiring metabolism for octanoic acid production. This result in conjunction with the results of other studies using OptForce in strain design demonstrates that this strategy may be also applicable to engineering E. coli for other customized bioproducts.

Keywords: Computational strain design, OptForce, CRISPR-Cas9, Octanoic acid (C8), Combinatorial engineering

\footnotetext{
*Correspondence: jshanks@iastate.edu

†Zaigao Tan and Jong Moon Yoon contributed equally to this work

${ }^{1}$ Department of Chemical and Biological Engineering, lowa State

University, 3031 Sweeney, Ames, IA 50011, USA

Full list of author information is available at the end of the article
} 


\section{Background}

The use of microbial biocatalysts for biorenewables production is a promising alternative $[1,2]$ to the nonrenewable oil-based option. Among these biorenewables, fatty acids have received significant attention due to their wide range of applications [3-5]. Fatty acids are directly used a food preservative $[4,5]$. They also serve as precursor for synthesis of biocompatible polymers (e.g., polyanhydrides) with low toxicity [6]. In addition to such direct uses, fatty acids can also be described as a chemical intermediate that can be catalytically upgraded to a broad range of chemicals and fuels [7-9]. For instance, fatty acids can be decarboxylated to alkanes as diesel fuel $[8,10]$, or deoxygenated to alpha-olefins for ethylene polymerization $[11,12]$. Furthermore, fatty acids can be converted to fatty acid methyl or ethyl esters (FAME/ FAEE), which have higher energy density and lower water solubility than the first-generation biofuel ethanol [13].

Among microbial production organisms, Escherichia coli is widely recognized as an excellent host strain for production of biorenewable chemicals or fuels due to its fast growth, sequenced genome, and genetic tractability [12]. A variety of reports have described the metabolic engineering of $E$. coli for production of free fatty acids with high titer and yield [14-19] (Table 1). According to the chain length, fatty acids can be classified as short-chain fatty acids (SCFA), with 6-10 carbons, or medium-chain fatty acids (MCFA), with 12-18 carbons [12]. Octanoic acid (C8) is a representative SCFA and has advantages over MCFA, as octanoic acid methyl or ethyl esters have lower freezing points and kinematic viscosities in contrast to saturated MCFAMEs or MCFAEEs, and thus can enhance fuel quality and have wider applications [20].

Although many efforts have been successful in improving microbial production of MCFA [8, 12, 13, 21-23], production of short-chain C8 is not as advanced. Recently, S. cerevisiae and mixed microbial communities have been engineered to produce octanoate; however, the yields were still relatively low $(<320 \mathrm{mg} / \mathrm{L})$ [24-28]. Moreover, to our knowledge, the highest titer in minimal medium of free $\mathrm{C} 8$ using $E$. coli engineered with its existing synthesis mechanisms is no more than $250 \mathrm{mg} / \mathrm{L}$ [17, 29, 30] (Table 1), which suggests that the existing metabolic network in $E$. coli is not optimal for C8 production. To this end, engineering of $E$. coli inherent metabolism to release its C8 synthesis capacity is desirable. However, engineering strategies that are useful for improving MCFA production might be not simply applied to improving SCFA production due to distinct acyl-ACP thioesterases and optimal rates of acyl-ACP elongation $[17,29]$. Although intuitive trial-and-error engineering may be ultimately successful, it is also labor intensive. In contrast, computational strain design tools, such as OptForce, have shown promise in selecting metabolic targets for production of desired targets, due to consideration of the complex interconnectivity of cellular metabolism [12, 31, 32].

OptForce is an optimization procedure to identify all genetic manipulations required for overproduction of targeted chemicals. It makes use of available flux measurements with a stoichiometric genome-scale model and extracts a minimal set of metabolic fluxes which must actively be forced through genetic manipulations (i.e., FORCE set) to make sure that all fluxes in the metabolic network are consistent with the overproduction objective [33]. Our prior study employed OptForce for prediction of metabolic targets for $\mathrm{C} 8$ production [31]. According to the OptForce prediction for $\mathrm{C} 8$ overproduction, four distinct interventions (FORCE sets) were suggested (Fig. 1). By using a rapid and iterative CRISPR-Cas9 technique [34], a variety of C8-producing E. coli strains were constructed. Among them, the combinatorial ZEFA (TE10) strain exhibits the best performance, resulting in a free C8 titer of $1 \mathrm{~g} / \mathrm{L}$ (90\% extracellular) and a high selectivity $(>70 \%)$ in $\mathrm{M} 9$ medium using glucose as the sole carbon source. These results demonstrate the effectiveness of combinatorial utilization of computational strain design and experimental approach for free $\mathrm{C} 8$ production.

\section{Results \\ Metabolic interventions suggested by OptForce for octanoic acid production}

The type II fatty acid biosynthesis (FAB) pathway is recognized as the primary route of fatty acid production by E. coli [35]. Figure 1 briefly illustrates the anabolic and catabolic metabolism of octanoic acid (C8) within E. coli. Acetyl-CoA serves as both initiation primer of fatty acid synthesis and precursor of malonyl-acyl carrier protein (ACP). Specifically, acetyl-CoA is used by acetyl-CoA carboxylase (ACC) to produce malonyl-CoA, which is subsequently converted to malonyl-ACP by malonylCoA-ACP transacylase (FabD). The initiation of fatty acid synthesis starts with condensation of acetyl-CoA and malonyl-ACP to form butyryl-ACP, which serves as the core for chain elongation. During each round of the elongation cycle, a two-carbon unit from malonyl-ACP is added to the fatty acyl-ACP chain. Finally, the elongated acyl-ACP is hydrolyzed by thioesterase to release free fatty acid. For production of free C8, the TE10 thioesterase from Anaerococcus tetradius [29], which primarily hydrolyzes octanoyl-ACP, was transformed into producer strains. Free octanoic acid (or octanoate anion) whose carboxyl group was not esterified with glycerol-3-phosphate to form lipids is found to be the predominant fatty acid (80-90\%) produced by strains harboring TE10 gene 
Table 1 Metabolic engineering of $E$. coli existing fatty acid biosynthesis pathway for fatty acid production in minimal medium

\begin{tabular}{|c|c|c|c|c|c|c|c|c|}
\hline Fatty acid & Strain & Genetic modifications & Thioesterase & Culture condition & Titer (g/L) & $\begin{array}{l}\text { Yield } \\
\text { (mg/g } \\
\text { glucose) }\end{array}$ & $\begin{array}{l}\text { Productivity } \\
\text { (mg/L/h) }\end{array}$ & Source \\
\hline \multirow[t]{7}{*}{ MCFA (C12-C18) } & BL21 & $\triangle f a d L$ & TesA & $\begin{array}{l}\text { Bioreactor, minimal } \\
\text { medium with } 2 \% \\
\text { (wt/v) glucose }\end{array}$ & 4.8 & 44 & 126 & {$[15]$} \\
\hline & BL21 & & AbTE & $\begin{array}{l}\text { Bioreactor, M9 medium } \\
\text { with } 0.5 \% \text { tryptone } \\
\text { and feeding of } \\
\text { glucose }\end{array}$ & 3.6 & 61 & 89 & [19] \\
\hline & BL21 & $\Delta f a d D,+a c c$ & TesA'+ CcTE & $\begin{array}{l}\text { Bioreactor, M9 with } \\
\text { feeding of glycerol }\end{array}$ & 2.5 & 48 & 170 & {$[16]$} \\
\hline & MG1655 & $\Delta f a d D,+f a b z$ & RcTE & $\begin{array}{l}\text { Shake flask, M9 with } \\
1.5 \% \text { glucose }\end{array}$ & 1.7 & 113 & 35 & {$[27]$} \\
\hline & $\mathrm{DH} 1$ & $\triangle f a d E$ & TesA' & $\begin{array}{l}\text { Shake flask, minimal } \\
\text { with } 2 \% \text { glucose }\end{array}$ & 3.8 & 190 & 53 & {$[50]$} \\
\hline & $\mathrm{DH} 1$ & $+f a d R$ & TesA & $\begin{array}{l}\text { Shake flask, minimal } \\
\text { with } 2 \% \text { glucose }\end{array}$ & 5.2 & 260 & 72 & {$[51]$} \\
\hline & BL21 & $\begin{array}{l}\text { Modular optimization of } \\
\text { multi-genes }\end{array}$ & CnFatB2 & $\begin{array}{l}\text { Bioreactor, MK with 1\% } \\
\text { YE and feeding of } \\
\text { glucose }\end{array}$ & 8.6 & 78 & 124 & [18] \\
\hline \multirow[t]{7}{*}{ Octanoate (C8) } & K27 & $\triangle f a d D$ & Various & $\begin{array}{l}\text { Culture tube, LB-grown } \\
\text { preculture was resus- } \\
\text { pended in M9 with } \\
0.4 \% \text { glucose }\end{array}$ & $0.18^{\mathrm{a}}$ & $48.0^{\mathrm{a}}$ & $10.0^{\mathrm{a}}$ & [24] \\
\hline & BL21 (DE3) & $\begin{array}{l}\triangle f a d D \triangle p t a \triangle l a c Y \text { fabF- } \\
\text { mut fabBDeg }\end{array}$ & CpFatB1 & $\begin{array}{l}\text { 96-well plate, LB-grown } \\
\text { preculture was diluted } \\
\text { 1:20 in M9 with 0.5\% } \\
\text { glucose }\end{array}$ & $0.24^{\mathrm{a}}$ & $45.0^{\mathrm{a}}$ & $4.5^{\mathrm{a}}$ & {$[17]$} \\
\hline & MG1655 & $\triangle f a d D$ & AtTE & $\begin{array}{l}\text { Bioreactor, MOPS with } \\
2 \% \text { glucose }\end{array}$ & 0.044 & 4.7 & 0.45 & {$[26]$} \\
\hline & MG1655 & $\begin{array}{l}\text { IdhA::M1-93-pssA, } \\
\text { mgsA::M1-93-acrAB, } \\
\text { maeB::M1-93-to/C }\end{array}$ & AtTE & $\begin{array}{l}\text { Shake flask, MOPS with } \\
2 \% \text { glucose }\end{array}$ & 0.22 & 20.0 & 3.1 & {$[25]$} \\
\hline & MG1655 & $\begin{array}{l}\text { mgsA::M1-93-fabZ } \triangle \text { fadE } \\
\quad \triangle f u m A C \triangle a c k A\end{array}$ & AtTE & $\begin{array}{l}\text { Shake flask, M9 with } \\
1.5 \% \text { glucose }\end{array}$ & 0.44 & 30.0 & 6.2 & This study \\
\hline & MG1655 & Same as above & AtTE & $\begin{array}{l}\text { Bioreactor, M9 with } \\
1.5 \% \text { glucose }\end{array}$ & 0.50 & 33.3 & 10.4 & This study \\
\hline & MG1655 & Same as above & AtTE & $\begin{array}{l}\text { Bioreactor, M9 with } \\
2.63 \% \text { glucose }\end{array}$ & 1.0 & 38.0 & 10.4 & This study \\
\hline
\end{tabular}

TE, thioesterase; TesA', cytosolic E. coli TE 1; FatB, plant fatty acyl-ACP thioesterase; Ab, Acinetobacter baylyi; Cc, Cinnamomum camphorum; Rc, Ricinus communis; Cn, Cocos nucifera; $\mathrm{Cp}$, Cuphea palustris; At, Anaerococcus tetradius; $\mathrm{YE}$, yeast extract

a Using LB as preculture

(Fig. 2), which is consistent with previous reports [29]. Conversely, free fatty acids can be also degraded by $E$. coli. Fatty acid is firstly acylated by fatty acyl-CoA synthetase (FadD) to form fatty acyl-CoA, which then enters into the $\beta$-oxidation cycle pathway for degradation. During each turn of the oxidation cycle, a two-carbon unit is removed from the acyl-CoA chain to produce one molecule of acetyl-CoA [35].

The OptForce algorithm was previously used to identify a prioritized set of metabolic interventions that would rewire the flux topology of native $E$. coli metabolism towards overproduction of octanoic acid [31, 33] (Fig. 1). The simulations, performed under aerobic glucose minimal medium conditions using the iAF1260 genome-scale metabolic model of $E$. coli (Additional file 1: Table S1) [31], suggested a set of four reaction-level manipulations that improve C 8 yield to almost $90 \%$ of its theoretical maximum [31, 33] (Fig. 1). The primary interventions include (i) up-regulating any of the fatty acid chain elongation reactions in the C8-chain, i.e., 3-oxyacyl-ACP synthase (3OAS80), 3-oxo-acyl-ACP reductase (3OAR80), or 3-hydroxy-acyl-ACP dehydratase 


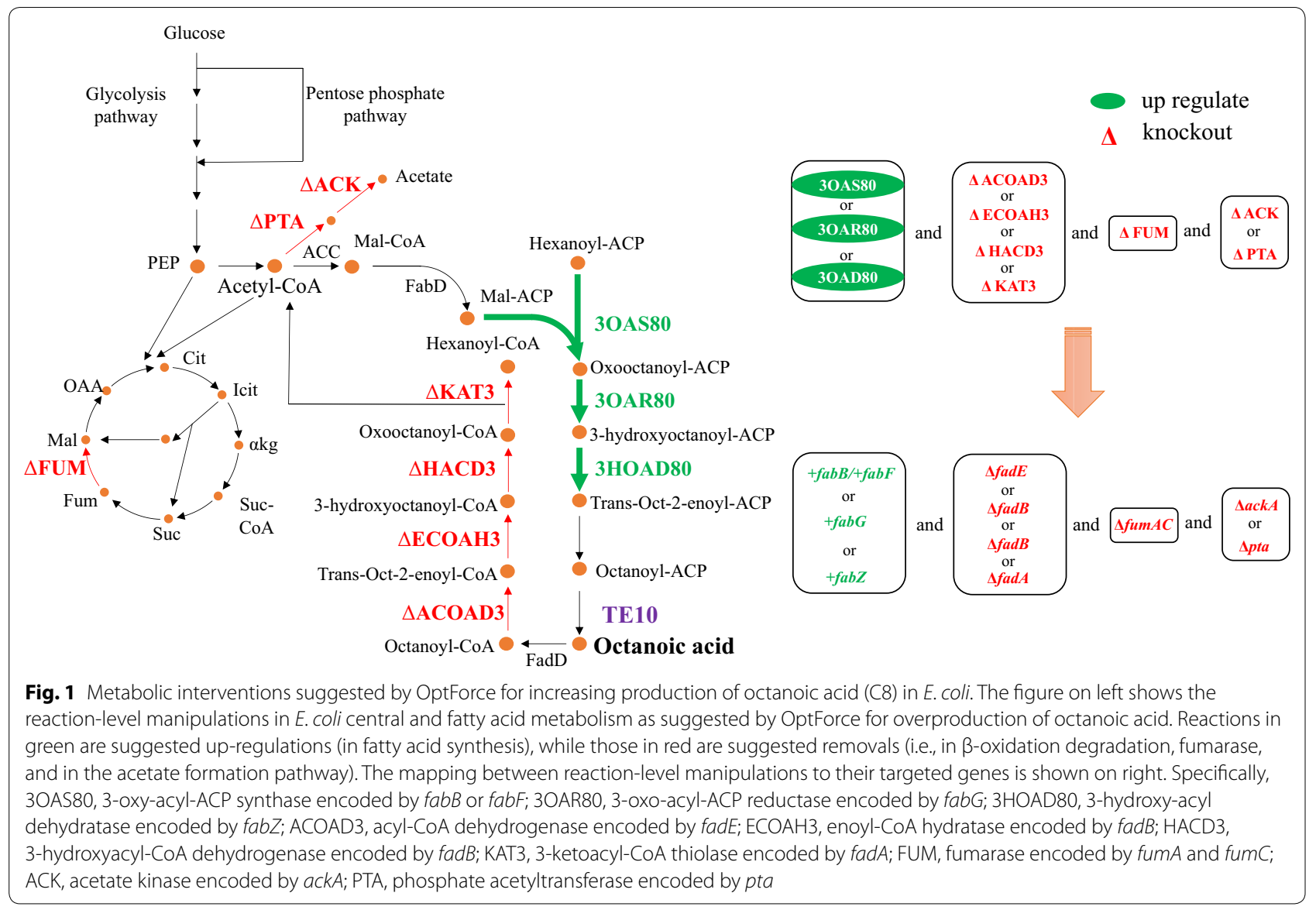

(3HAD80) by at least two times of the maximum achievable flux in the wild-type E. coli; (ii) elimination of any reactions in the $\beta$-oxidation pathway, i.e., acyl-CoA dehydrogenase (ACOAD3), enoyl-CoA hydratase (ECOAH3), 3-hydroxyacyl-CoA dehydrogenase (HACD3), 3-ketoacyl-CoA thiolase (KAT3). Together, these two interventions account for over $86 \%$ of the predicted C8 yield increase [31].

Besides being the precursor for fatty acid synthesis, acetyl-CoA also has two additional roles. First, acetyl$\mathrm{CoA}$ and oxaloacetic acid (OAA) can be catalyzed by citrate synthase to form citrate as part of the TCA cycle [32]. Consequently, OptForce suggests elimination of fumarase (FUM) to disrupt the TCA cycle activity and maintain a higher pool of acetyl-CoA to be redirected towards the fatty acid elongation. In addition, acetyl-CoA can also be converted by phosphotransacetylase (PTA) and acetate kinase (ACK) to acetate. Therefore, OptForce targets elimination of the acetate formation pathway (Fig. 1). Overall, the flux redirection strategy suggested by OptForce could be summarized as up-regulation of the target pathway (i.e., the C8 chain of fatty acid synthesis), followed by elimination of competing paths that either degrade the product (i.e., the $\beta$-oxidation pathway) or degrade the precursor (i.e., the TCA and acetate synthesis pathway). Note that OptForce predictions are based on a stoichiometry-only model of E. coli metabolism, and do not include other significant factors, such as enzyme kinetics and transcriptional and substrate-level regulation into consideration. Therefore, the interventions and target yields predicted by OptForce should be considered as a starting point for rewiring the metabolism, rather than guaranteed hits.

\section{Effects of individual interventions on octanoic acid production}

As shown in Fig. 1, interventions suggested by OptForce are at the level of metabolic reactions. In order to implement these interventions genetically, it is essential to translate the "reaction" language into "gene" language [32]. Within the annotated genome of E. coli MG1655, we identified a total of 10 genes encoding enzymes for these reactions (Fig. 1). These 10 genes can be divided into four different modules: (i) fatty acids biosynthesis (Fab), including $f a b B, f a b F, f a b G$, and $f a b Z$ genes; (ii) fatty acids degradation (Fad), including $f a d E$, $f a d A$, and $f a d B$ genes; 

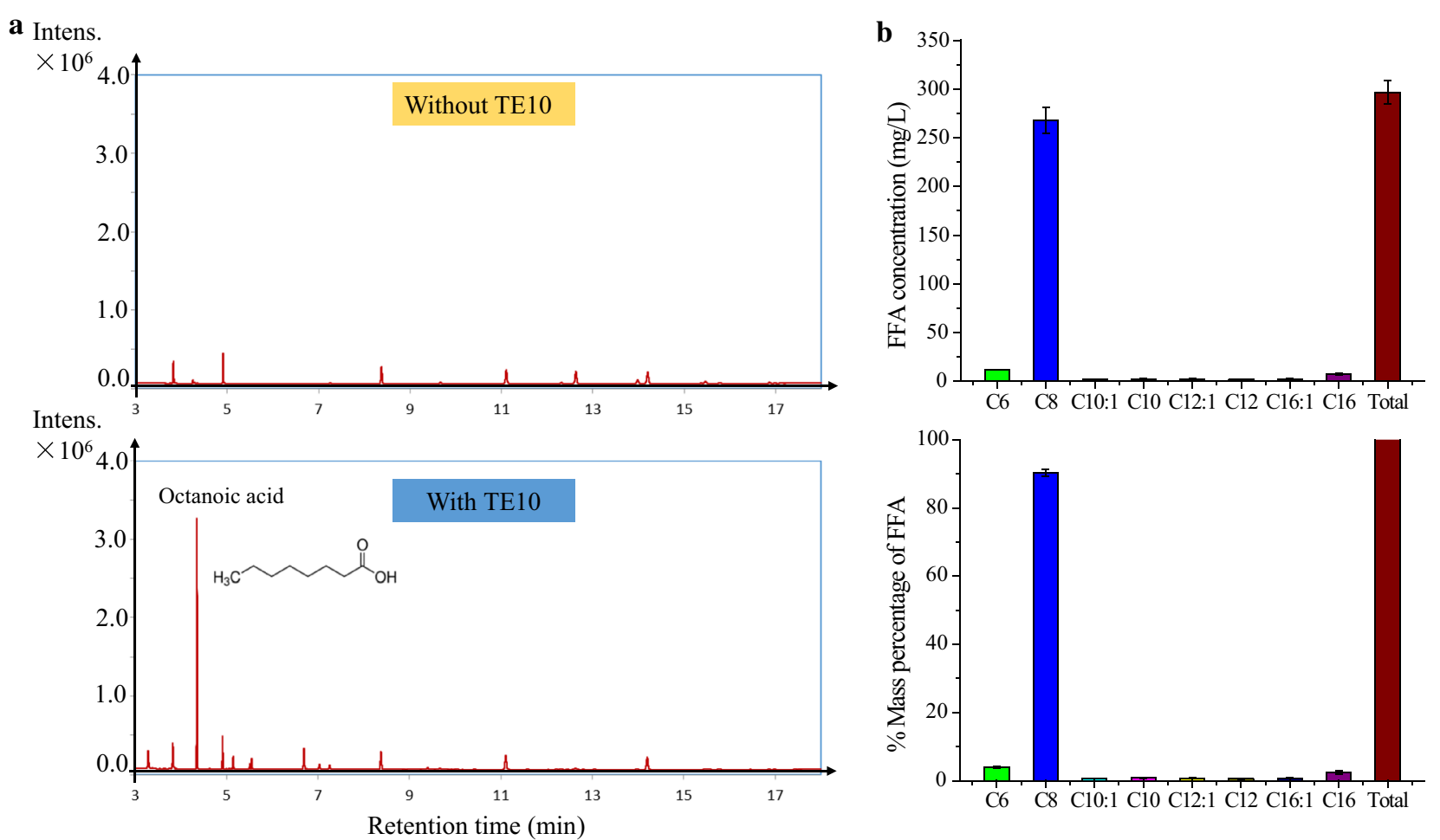

Fig. 2 Fatty acids production in E. coli strain harboring TE10. a Fatty acids profile of E. coli MG1655 strains without (upper) and with (lower) pJMYEEI82564 plasmid carrying thioesterase TE10 from Anaerococcus tetradius. b FFAs concentration and distribution in E. coli strain MG1655 harboring TE10. Upper, final free fatty acid titers at $72 \mathrm{~h}$; lower, $\%$ mass percentage of produced fatty acids. Cultures were performed in $40 \mathrm{~mL} \mathrm{M9}+1.5 \%$ (wt/v) dextrose in $250-\mathrm{mL}$ shake flasks at $250 \mathrm{rpm} 30^{\circ} \mathrm{C}$ with an initial pH of 7.0, IPTG of $1 \mathrm{mM}$. Titers are the average of at least three biological replicates at $72 \mathrm{~h}$ with error bars indicating one standard deviation. TE10, thioesterase from Anaerococcus tetradius

(iii) FUM, including fumAC genes, and (iv) acetate (Ace), including ack $A$ and $p t a$ genes.

The first set of prioritized interventions suggested by OptForce for production of $\mathrm{C} 8$ includes up-regulation of one of the chain elongation reactions in the Fab module (Fig. 1). To this end, the effect of increased expression of individual $f a b$ genes on $\mathrm{C} 8$ production was investigated. Prior research has demonstrated that overexpression of pathway genes might cause metabolic burdens, such as from plasmid maintenance, leading to a trade-off in production [36]. To this end, here we overexpressed the $f a b$ genes by inserting a second copy of $f a b$ gene into genomic DNA of $E$. coli MG1655 at the $m g s A$ site, with regulated by a strong constitutive promoter M1-93 [30]. Increasing the expression of $f a b Z$, which encodes the 3-hydroxyacylACP dehydratase, significantly increased $\mathrm{C} 8$ production (Table 2). Specifically, $+f a b Z$ (TE10) strain produced 398 (26.9 mg/g glucose, yield) and $479 \mathrm{mg} / \mathrm{L}$ of free C8 and total FAs, which exceeded the wild-type MG1655 (TE10) production of $275 \mathrm{mg} / \mathrm{L}$ ( $19.9 \mathrm{mg} / \mathrm{g}$ glucose) free C8 and $315 \mathrm{mg} / \mathrm{L}$ total FAs by more than 45 and $52 \%$, respectively $(P<0.01)$. Increased expression of the $f a b G$ gene, encoding the 3-oxo-acyl-ACP reductase, led to no significant change in C8 production $(P>0.05)$ (Table 2). Unexpectedly, increased expression of some $f a b$ genes resulted in decreased $\mathrm{C} 8$ production. For instance, $+f a b B$ (3-oxy-acyl-ACP synthase I) (TE10) or $+f a b F$ (3-oxy-acyl-ACP synthase II) (TE10) strains each produced roughly $250 \mathrm{mg} / \mathrm{L}$ of ( $19.0 \mathrm{mg} / \mathrm{g}$ glucose) C 8 and $285 \mathrm{mg} / \mathrm{L}$ of total FAs, an approximately $10 \%$ decrease relative to the starting strain MG1655 (TE10) $(P<0.05)$. While this result is in conflict with the OptForce predictions, which is based only upon stoichiometric considerations, it is consistent with prior observations that inhibiting the activity of FabB and FabF contributed to increased production of $\mathrm{C} 8$, likely through regulatory effects [17]. From metabolic control analysis, linear pathway enzymes may share control for the flux through that pathway, and thus genes for all enzymes in the metabolic pathway should be overexpressed together for further increasing the production of desired targets [37-41]. To this end, $+f a b G$ and $+f a b B / F$ based on $+f a b Z$ was performed $(+f a b Z G B /+f a b Z G F$, Additional file 2: Figure S1). However, compared with $+f a b Z$, both $+f a b Z G B$ and $+f a b Z G F$ decreased rather than increased $C 8$ production (Table 2). 
Table 2 Effects of engineering individual genes on free octanoic acid production

\begin{tabular}{|c|c|c|c|c|c|c|c|c|}
\hline Strain & Time (h) & $\begin{array}{l}\text { Cell density } \\
\left(\mathrm{OD}_{550}\right)\end{array}$ & $\begin{array}{l}\text { Glucose used } \\
\text { (g/L) }\end{array}$ & $\begin{array}{l}\text { Acetate titer } \\
(\mathbf{g} / \mathbf{L})\end{array}$ & $\begin{array}{l}\text { C8 titer } \\
\text { (mg/L) }\end{array}$ & $\begin{array}{l}\text { C8 yield ( } \mathrm{mg} / \mathrm{g} \\
\text { glucose) }\end{array}$ & $\begin{array}{l}\text { C8 productivity } \\
(\mathrm{mg} / \mathrm{L} / \mathrm{h})\end{array}$ & $\begin{array}{l}\text { Total FFAs } \\
(\mathrm{mg} / \mathrm{L})\end{array}$ \\
\hline MG1655 (TE10) & 72 & $1.91 \pm 0.1$ & $13.8 \pm 0.2$ & $0.63 \pm 0.02$ & $275 \pm 12$ & $19.9 \pm 0.9$ & $3.81 \pm 0.17$ & $315 \pm 14$ \\
\hline$+f a b B(T E 10)$ & 72 & $1.87 \pm 0.06$ & $13.4 \pm 0.1$ & $0.62 \pm 0.02$ & $250 \pm 5.7$ & $18.6 \pm 0.4$ & $3.46 \pm 0.079$ & $281 \pm 5.7$ \\
\hline +fabF (TE10) & 72 & $1.72 \pm 0.05$ & $13.2 \pm 0.3$ & $0.65 \pm 0.03$ & $253 \pm 6.4$ & $19.2 \pm 0.3$ & $3.52 \pm 0.088$ & $292 \pm 11$ \\
\hline +fabG (TE10) & 72 & $1.81 \pm 0.1$ & $13.9 \pm 0.4$ & $0.67 \pm 0.05$ & $293 \pm 5.5$ & $21.0 \pm 0.4$ & $4.07 \pm 0.076$ & $326 \pm 28$ \\
\hline$+f a b Z$ (TE10) & 72 & $4.51 \pm 0.2$ & $14.8 \pm 0.1$ & $1.8 \pm 0.2$ & $398 \pm 8.3$ & $26.9 \pm 0.6$ & $5.53 \pm 0.12$ & $479 \pm 22$ \\
\hline$+f a b Z G B(T E 10)$ & 72 & $5.74 \pm 0.4$ & $14.8 \pm 0.1$ & $0.52 \pm 0.06$ & $278 \pm 13$ & $18.8 \pm 0.9$ & $3.86 \pm 0.18$ & $403 \pm 16$ \\
\hline$+f a b Z G F(T E 10)$ & 72 & $5.50 \pm 0.2$ & $14.6 \pm 0.1$ & $1.1 \pm 0.2$ & $207 \pm 9.5$ & $14.2 \pm 0.7$ & $2.88 \pm 0.13$ & $309 \pm 11$ \\
\hline$\triangle$ fadE (TE10) & 72 & $2.20 \pm 0.1$ & $13.6 \pm 0.4$ & $1.2 \pm 0.1$ & $286 \pm 8.8$ & $21.1 \pm 0.6$ & $3.97 \pm 0.12$ & $333 \pm 10$ \\
\hline$\triangle f a d B(T E 10)$ & 72 & $1.84 \pm 0.1$ & $13.3 \pm 0.1$ & $1.3 \pm 0.1$ & $270 \pm 4.4$ & $20.3 \pm 0.3$ & $3.75 \pm 0.062$ & $310 \pm 5.2$ \\
\hline$\triangle$ fadA (TE10) & 72 & $1.84 \pm 0.02$ & $13.8 \pm 0.2$ & $1.4 \pm 0.1$ & $270 \pm 3.5$ & $19.5 \pm 0.3$ & $3.74 \pm 0.048$ & $309 \pm 4.4$ \\
\hline$\triangle$ fumAC (TE10) & 72 & $1.73 \pm 0.07$ & $12.9 \pm 0.3$ & $1.4 \pm 0.2$ & $241 \pm 6.5$ & $18.7 \pm 0.5$ & $3.35 \pm 0.048$ & $277 \pm 3.9$ \\
\hline$\triangle a c k A(T E 10)$ & 72 & $2.06 \pm 0.06$ & $13.2 \pm 0.1$ & $0.6 \pm 0.1$ & $289 \pm 5.7$ & $21.9 \pm 0.4$ & $4.00 \pm 0.079$ & $330 \pm 6.6$ \\
\hline$\Delta p t a($ TE10) & 72 & $1.97 \pm 0.03$ & $12.9 \pm 0.2$ & $0.5 \pm 0.1$ & $284 \pm 2.6$ & $22.0 \pm 0.2$ & $3.94 \pm 0.036$ & $324 \pm 3.4$ \\
\hline
\end{tabular}

In this study, the genetic interventions identified by OptForce are prioritized based on their impact on C8 product yield improvement. In addition to the first set, the second set of prioritized interventions predicted by OptForce is interruption of the $\beta$-oxidation cycle. In contrast to the first set of Fab module, engineering of the corresponding Fad module by deletion of any fadE (acylCoA dehydrogenase), fadA (3-ketoacyl-CoA thiolase), or $\mathrm{fadB}$ (enoyl-CoA hydratase/3-hydroxyacyl-CoA dehydrogenase) led to no significant change in production of $\mathrm{C} 8$ or total FAs $(P>0.05)$ (Table 2).

The third set of prioritized interventions suggested by OptForce is to remove fumarase (FUM) in the TCA cycle in order to maintain higher acetyl-CoA pool towards the $\mathrm{C} 8$ elongation chain. $E$. coli has three distinct fumarase isozymes, encoded by fumA, fumB, and fum $C$ [42]. Unlike fumB, the fum $A$ and fum $C$ genes are expressed primarily under aerobic conditions [43]. Therefore, fumA and $f u m C$ were selected as targets for disruption of aerobic fumarase activity. Since fumA and fum $C$ are cotranscribed, the two genes were deleted simultaneously, resulting in $\triangle f u m A C$ strain. Results showed that $\triangle f u m A C$ (TE10) decreased free $\mathrm{C} 8$ and total FAs production by $12 \%$ to $241 \mathrm{mg} / \mathrm{L}(18.7 \mathrm{mg} / \mathrm{g}$ glucose $)$ and $277 \mathrm{mg} / \mathrm{L}$ relative to the starting strain MG1655 (TE10) $(P<0.01)$ (Table 2). The fourth set of prioritized interventions involves removal of phosphotransacetylase (PTA) and acetate kinase $(\mathrm{ACK})$ in the acetate formation pathway (Ace module). Specifically, individual deletion of $a c k A$ or pta resulted in limited change in free C8 or total FAs production (Table 2), which is consistent with the prior observation of deletion effects on long-chain fatty acid production [44].
Taken together, among these interventions, engineering of the Fab module, especially the up-regulation of $f a b Z$ ( $+f a b Z$ ), imposed the highest improvement in $\mathrm{C} 8$ production, which is in line with the prioritized order suggested by OptForce.

\section{Combinatorial utilization of the suggested four interventions}

While there are cases where individual interventions have improved the target yield [32], in this case for C8, OptForce suggests these interventions should be examined in their prioritized order. Actually, effects of modules with less priority such as FUM, Ace should be seen in conjunction with modules with high priority, such as Fab and Fad modules. Therefore, after knowing the effect of individual interventions on $\mathrm{C} 8$ production, combinatorial utilization of interventions was performed with the aim of further C8 improvement, and the CRISPR-Cas9 method, which has the advantages of iterative genome engineering, was further employed to construct these combinatorial strains [34].

As the $+f a b Z$ intervention in the Fab module enabled the highest increase in C8 production $(+45 \%)$ (Table 2; Fig. 3), it was selected as the representative of the Fab module for subsequent implementation of other interventions. The suggested genes in the Fad module ( $\mathrm{fadE}$, $f a d B, f a d A$ ) were then individually deleted from the $+f a b Z$ (TE10) strain. However, no significant increase was observed in free C8 production (Table 3). Since $+f a b Z \triangle f a d E$ (TE10) was one of the best-performing strains after $+f a b Z \Delta f a d$ engineering, it was therefore selected for the next round of FUM module engineering. 


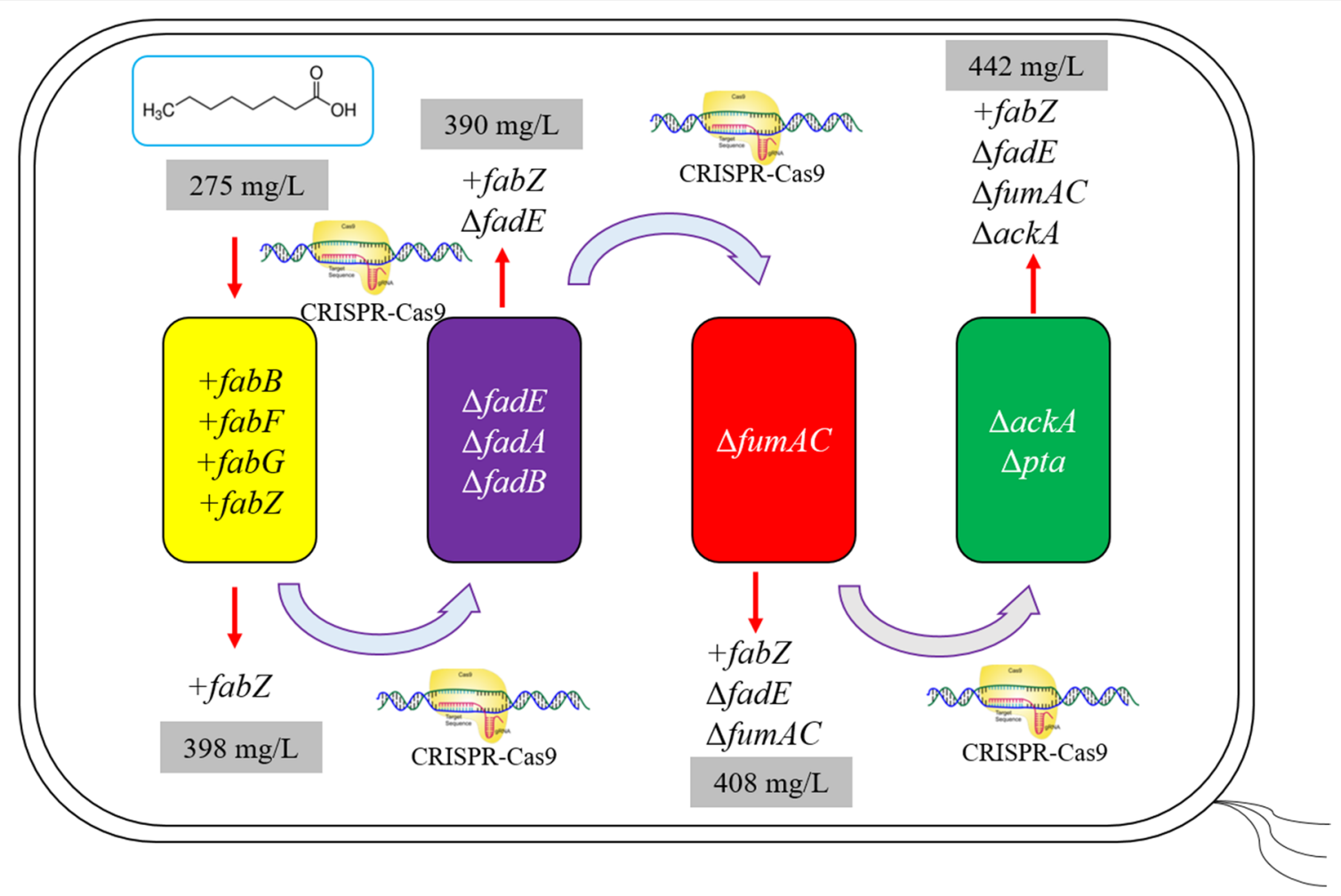

Fig. 3 Schematic of combinatorial utilization of different interventions for octanoic acid production. CRISPR-Cas9 technique [34] was employed to construct these combinatorial engineered strains

Table 3 Effects of combinatorial implementation of different interventions on free octanoic acid production

\begin{tabular}{|c|c|c|c|c|c|c|c|c|}
\hline Strain & Time (h) & $\begin{array}{l}\text { Cell density } \\
\left(\mathrm{OD}_{550}\right)\end{array}$ & $\begin{array}{l}\text { Glucose used } \\
\text { (g/L) }\end{array}$ & $\begin{array}{l}\text { Acetate titer } \\
\text { (g/L) }\end{array}$ & $\begin{array}{l}\text { C8 titer } \\
\text { (mg/L) }\end{array}$ & $\begin{array}{l}\text { C8 yield ( } \mathrm{mg} / \mathrm{g} \\
\text { glucose) }\end{array}$ & $\begin{array}{l}\text { C8 productivity } \\
\text { (mg/L/h) }\end{array}$ & $\begin{array}{l}\text { Total FFAs } \\
\text { (mg/L) }\end{array}$ \\
\hline MG1655 (TE10) & 72 & $1.91 \pm 0.1$ & $13.8 \pm 0.2$ & $0.63 \pm 0.02$ & $275 \pm 12$ & $19.9 \pm 0.9$ & $3.81 \pm 0.17$ & $315 \pm 14$ \\
\hline$+f a b Z(T E 10)$ & 72 & $4.51 \pm 0.2$ & $14.8 \pm 0.1$ & $1.8 \pm 0.2$ & $398 \pm 8.3$ & $26.9 \pm 0.6$ & $5.53 \pm 0.12$ & $479 \pm 22$ \\
\hline $\begin{array}{l}+f a b Z \triangle f a d E \\
(T E 10)\end{array}$ & 72 & $4.62 \pm 0.1$ & $14.8 \pm 0.1$ & $1.7 \pm 0.02$ & $387 \pm 12$ & $26.2 \pm 0.8$ & $5.38 \pm 0.16$ & $462 \pm 14$ \\
\hline $\begin{array}{l}+f a b Z \Delta f a d B \\
(\text { TE10) }\end{array}$ & 72 & $4.76 \pm 0.3$ & $14.8 \pm 0.1$ & $1.3 \pm 0.3$ & $395 \pm 8.2$ & $26.7 \pm 0.5$ & $5.49 \pm 0.11$ & $469 \pm 12$ \\
\hline $\begin{array}{l}+f a b Z \triangle f a d A \\
(T E 10)\end{array}$ & 72 & $1.90 \pm 0.1$ & $13.2 \pm 0.4$ & $0.9 \pm 0.2$ & $300 \pm 5.7$ & $22.7 \pm 0.4$ & $4.17 \pm 0.079$ & $350 \pm 6.2$ \\
\hline $\begin{array}{l}+ \text { fabZ } \triangle f a d E \\
\quad \triangle f u m A C(T E 10)\end{array}$ & 72 & $4.74 \pm 0.2$ & $14.4 \pm 0.2$ & $1.1 \pm 0.2$ & $408 \pm 4.8$ & $28.3 \pm 0.3$ & $5.67 \pm 0.066$ & $515 \pm 8.8$ \\
\hline $\begin{array}{l}+f a b Z \triangle f a d E \\
\quad \triangle f u m A C \triangle a c k A \\
\text { (TE10) }\end{array}$ & 72 & $5.38 \pm 0.1$ & $14.8 \pm 0.1$ & $1.4 \pm 0.1$ & $442 \pm 14$ & $30.0 \pm 0.8$ & $6.15 \pm 0.19$ & $615 \pm 21$ \\
\hline $\begin{array}{l}+f a b Z \triangle f a d E \\
\quad \triangle f u m A C \triangle p t a \\
\text { (TE10) }\end{array}$ & 72 & $4.79 \pm 0.03$ & $14.6 \pm 0.1$ & $1.4 \pm 0.3$ & $408 \pm 4.9$ & $27.2 \pm 0.3$ & $5.67 \pm 0.068$ & $532 \pm 5.9$ \\
\hline
\end{tabular}

In contrast to the decreased $\mathrm{C} 8$ production by the $\triangle$ fumAC (TE10) strain, disruption of fumAC in the $+f a b Z \triangle f a d E$ (TE10) strain improved free C8 production (Table 3). Specifically, the triple $+f a b Z \Delta f a d E \Delta f u m A C$ (TE10) produced $408 \mathrm{mg} / \mathrm{L}$ of C8 (28.3 mg/g glucose) and
$515 \mathrm{mg} / \mathrm{L}$ of total FAs, which exceeds that of $+f a b Z \Delta f a d E$ (TE10) strain $(\mathrm{C} 8=387 \mathrm{mg} / \mathrm{L}, 26.2 \mathrm{mg} / \mathrm{g}$ glucose; total FAs $=462 \mathrm{mg} / \mathrm{L})$ by 5.4 and $11.5 \%$, respectively $(P=0.04)$. This observation is in line with OptForce predictions that suggest an increase in octanoic acid yield due to 
removal of fumarase activity only in the background of manipulations in the fatty acid synthesis and degradation pathways. This $+f a b Z \Delta f a d E \triangle f u m A C$ (TE10) strain was used for the next round engineering of Ace module engineering. Although deletion of $p t a$ in $+f a b Z \Delta f a d E$ $\triangle$ fumAC (TE10) did not further increase free C8 production, deletion of ack $A$ did substantially improve C8 production ( $P=0.009)$ (Fig. 3). Specifically, the resulting quadruple strain +fabZ $\triangle$ fadE $\triangle$ fumAC $\triangle a c k A$ (TE10) (ZEFA (TE10)) produced $442 \mathrm{mg} / \mathrm{L}$ of $\mathrm{C} 8(30.0 \mathrm{mg} / \mathrm{g}$ glucose) and $615 \mathrm{mg} / \mathrm{L}$ of total FAs, which exceeded that of $+f a b Z \triangle f a d E \triangle f u m A C$ (TE10) by approximately 10 and $20 \%(C 8=408 \mathrm{mg} / \mathrm{L}, 28.3 \mathrm{mg} / \mathrm{g}$ glucose; total FAs $=515 \mathrm{mg} / \mathrm{L})($ Table 3; Fig. 3). Compared with the starting strain $+f a b Z$ (TE10) $(\mathrm{C} 8=398 \mathrm{mg} / \mathrm{L}, 26.9 \mathrm{mg} / \mathrm{g}$ glucose; total FAs $=479 \mathrm{mg} / \mathrm{L}$ ), ZEFA (TE10) produced 11 and $28 \%$ more $\mathrm{C} 8$ and total FAs $(P=0.002)$. Compared with the wild-type MG1655 (TE10) $(\mathrm{C} 8=275 \mathrm{mg} / \mathrm{L}$, $19.9 \mathrm{mg} / \mathrm{g}$ glucose; total FAs $=315 \mathrm{mg} / \mathrm{L}$ ), ZEFA (TE10) produced 61 and $95 \%$ more C 8 and total FAs $(P<0.001)$ (Table 3; Fig. 3). Similar to deletion of $\triangle$ fumAC, this result also highlights the prioritized intervention strategy suggested by OptForce for overproduction of octanoic acid.

\section{Optimization of culture conditions for octanoic acid production}

In this study, gene for encoding thioesterase TE10 is expressed under the control of trc promoter, and thus IPTG serves as inducer for expression of TE10 gene and $\mathrm{C} 8$ production. However, although useful, excessive IPTG addition has been reported to be toxic to E. coli cells [45], resulting in inhibition of enzymatic activities and decreased product biosynthesis [46]. To this end, optimization of IPTG dosage for fatty acid production is desirable [47]. Since ZEFA (TE10) is the best-performing C8-producing strain obtained in this study, it was selected for IPTG dosage optimization. Results showed that the ZEFA (TE10) strain can still produce $117 \mathrm{mg} / \mathrm{L}$ of free C8 without addition of IPTG, which means there is leaky expression of TE10 gene under the trc promoter (Fig. 4a). Upon induction by IPTG, C8 titer elevated significantly and a positive correlation was observed between IPTG dosage and C8 titer up to $200 \mu \mathrm{M}$. At the dosage of $200 \mu \mathrm{M}$ IPTG, ZEFA (TE10) strain produced $430 \mathrm{mg} / \mathrm{L}$ of $\mathrm{C} 8$ and $678 \mathrm{mg} / \mathrm{L}$ of total FAs, which was 3.7- and 2.4-fold of the non-IPTG condition (Fig. 4a). However, excessive dosage of IPTG, e.g., $>200 \mu \mathrm{M}$, was found to decrease $\mathrm{C} 8$ production (Fig. 4a).

The carbon-to-nitrogen $(\mathrm{C} / \mathrm{N})$ ratio has been observed to affect the production of biomass and fatty acid production in some microorganisms [48]. In order to investigate the impact of the $\mathrm{C} / \mathrm{N}$ ratio on free $\mathrm{C} 8$ production in the
ZEFA (TE10) strain, we varied the initial $\mathrm{C} / \mathrm{N}$ ratio in the M9 medium from 6.25 to 75.0 (Fig. 4b). Results showed that ZEFA (TE10) produced the highest C8 titer of 466 and $730 \mathrm{mg} / \mathrm{L}$ of total FAs $(\mathrm{IPTG}=200 \mu \mathrm{M})$ at the $\mathrm{C} / \mathrm{N}$ ratio of 18.8, and either lower or higher $\mathrm{C} / \mathrm{N}$ ratio was found to compromise $\mathrm{C} 8$ production (Fig. $4 \mathrm{~b}$ ).

In addition, we also found that medium acidification might be another limiting factor for free $\mathrm{C} 8$ production. For instance, the final pH of ZEFA (TE10) culture broth in shake flasks at $72 \mathrm{~h}$ was approximately 5.5 (data not shown). Broth acidification can inflict detrimental effects to E. coli growth and activities of key metabolic enzymes [49]. Maintenance of $\mathrm{pH}$ at neutral range is expected to mitigate the acidification burden and thus possibly improve $\mathrm{C} 8$ production. To this end, a $\mathrm{pH}$-controlled bioreactor, instead of shake flasks, was employed. With this $\mathrm{pH}$ control, the ZEFA (TE10) strain produced $500 \mathrm{mg} / \mathrm{L}$ of $\mathrm{C} 8$ and $805 \mathrm{mg} / \mathrm{L}$ of total FAs $(\mathrm{pH}=7.0$, IPTG $=200 \mu \mathrm{M}$ ), which is a 7.3 and $10.3 \%$ increase relative to shake flasks $(\mathrm{C} 8=466 \mathrm{mg} / \mathrm{L} ; \mathrm{TFA}=730 \mathrm{mg} / \mathrm{L})$ $(P<0.05)$ (Fig. 4c), and a 82 and $155 \%$ increase relative to wild-type MG1655 (TE10) in shake flasks $(\mathrm{C} 8=275 \mathrm{mg} / \mathrm{L}$; total FAs $=315 \mathrm{mg} / \mathrm{L})(P<0.01)$.

\section{Fed-batch culture of strain ZEFA (TE10) for octanoic acid production}

In this study, M9 medium with $1.5 \%(\mathrm{wt} / \mathrm{v})$ glucose was used as culture broth for free $\mathrm{C} 8$ production. For the ZEFA (TE10) strain, we found that there was no residual glucose after 72 -h cultivation in shake flasks (data not shown). Since glucose is the substrate, the provisioning of additional glucose may increase $\mathrm{C} 8$ titers. To this end, fed-batch culture was performed to the ZEFA (TE10) strain with previously described optimized culture conditions, i.e., $\mathrm{pH}$ maintained at 7.0, IPTG added at $200 \mu \mathrm{M}$, and the initial $\mathrm{C} / \mathrm{N}$ ratio kept at 18.8. Initially, $15 \mathrm{~g} / \mathrm{L}$ of glucose was used as carbon source and another $15 \mathrm{~g} / \mathrm{L}$ of fresh glucose was fed after 48-h cultivation (Fig. 5). Results showed that cell growth of the ZEFA (TE10) reached stationary phase at $24 \mathrm{~h}$, and the final highest optical density $\left(\mathrm{OD}_{550}\right)$ achieved 5.18 at $48 \mathrm{~h}$. The total culture process lasted for $120 \mathrm{~h}$ and the ZEFA (TE10) consumed a total of $26.3 \mathrm{~g} / \mathrm{L}$ of glucose, produced approximately $1 \mathrm{~g} / \mathrm{L}$ of free C8 $(38.5 \mathrm{mg} / \mathrm{g}$ glucose, $11 \%$ maximum theoretical yield) and $1.4 \mathrm{~g} / \mathrm{L}$ of total FAs (Fig. 5). Furthermore, over $90 \%$ of the C8 is recovered in the medium (data not shown). To our knowledge, this is the highest free $\mathrm{C} 8$ titer achieved in minimal medium using $E$. coli inherent fatty acid biosynthesis pathway. 

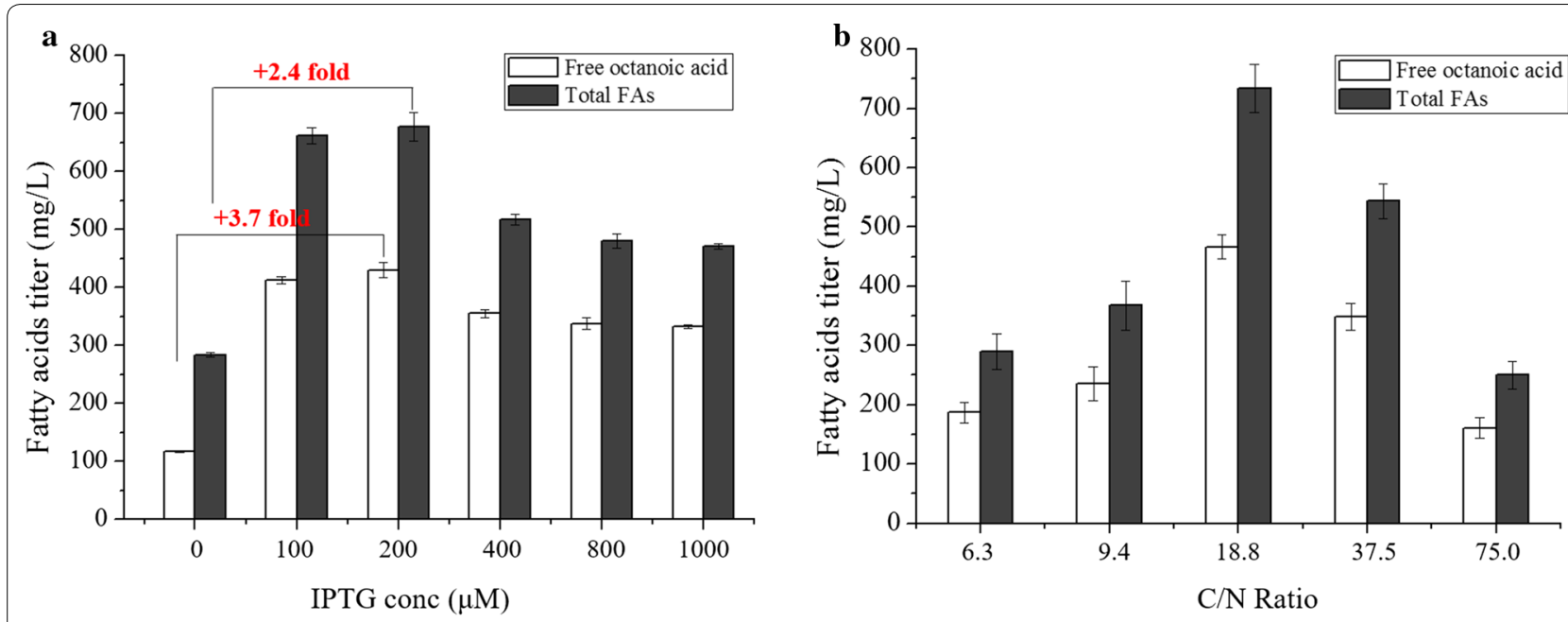

c

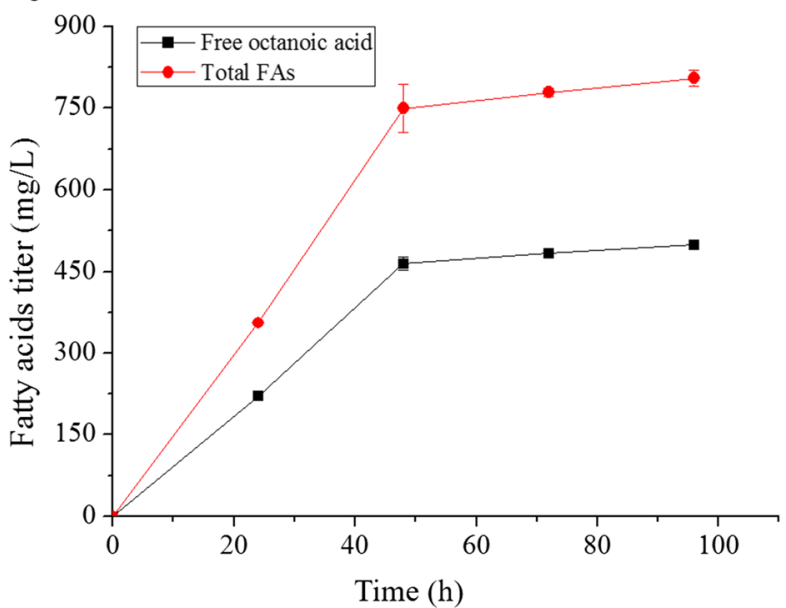

Fig. 4 Optimization of culture conditions for free octanoic acid production in the ZEFA (TE10) strain. a Effects of different IPTG dosages on octanoic acid production. ZEFA (TE10) strain produced the highest titer of octanoic acid (430 mg/L) when induced with $200 \mu \mathrm{M}$ IPTG for increasing expression of thioesterase TE10 gene. Cultures were performed in $40 \mathrm{~mL} \mathrm{M9+1.5 \%} \mathrm{(wt/v)} \mathrm{dextrose} \mathrm{with} \mathrm{the} \mathrm{C/N} \mathrm{ratio} \mathrm{of} 18.8$ in $250-\mathrm{mL}$ shake flasks at $250 \mathrm{rpm} 30^{\circ} \mathrm{C}$ with an initial pH of 7.0 and the IPTG was added when the OD ${ }_{550}$ reached $0.4-0.5$. $\mathbf{b}$ Effects of different initial C/N ratios on octanoic acid production under 200 MM IPTG. ZEFA (TE10) strain has the highest C8 production at the C/N ratio of 18.8. For changing the C/N ratio, the glucose (carbon source) concentration is fixed at $15 \mathrm{~g} / \mathrm{L}$ and the amount of $\mathrm{NH}_{4} \mathrm{Cl}$ (carbon source) added was varied accordingly. (C) Maintenance of culture broth $\mathrm{pH}$ at neutral range $(\mathrm{pH}=7.0)$ increased octanoic acid production under $200 \mu \mathrm{M}$ IPTG and C/N ratio of 18.8. Culture was performed in $300 \mathrm{~mL} \mathrm{M9+1.5 \%} \mathrm{(wt/v)} \mathrm{dextrose} \mathrm{in} \mathrm{a} 500-\mathrm{mL}$ bioreactor. Cultures were grown at $30^{\circ} \mathrm{C}$, and the pH was maintained at 7.0 by adding $2.0 \mathrm{M}$ potassium hydroxide $(\mathrm{KOH})$. Air flow rate was controlled at $0.3 \mathrm{~L} / \mathrm{min}$ with $300 \mathrm{rpm}$ as the initial stirring speed. The dissolved oxygen (DO) level was set over $40 \%$ and controlled by changing the stirring speed with the maximum of $600 \mathrm{rpm}$. Titers are the average of at least three replicates with error bars indicating one standard deviation. ZEFA, fadZ $\triangle$ fadE $\triangle$ fumAC $\triangle a c k A$

\section{Discussion}

In this study, we systematically engineered $E$. coli inherent metabolism network for overproduction of free C8. Different from previous strategies, this work focuses on exploiting rational computational design to identify the metabolic targets. Finally, a total of ten enzymes in four different interventions (or modules) are suggested by OptForce to be manipulated production. Here we found the effects of manipulations on $\mathrm{C} 8$ production are in line well with OptForce predictions of prioritizing orders.
Specifically, up-regulation of the Fab module is suggested to impose the most promising effect on $\mathrm{C} 8$ yield improvement and the experimental result confirmed this conclusion: increased expression of $f a b Z$ enabled the highest increase of $\mathrm{C} 8$ titer $(+45 \%)$ in $+f a b Z$ (TE10) engineered strain. Although this study focuses on $\mathrm{C} 8$ production, the potential positive effect of $+f a b Z$ intervention was also observed in other cases, such as MCFA (C14-C16) production in E. coli [23]. In addition, in vitro titration experiment also showed that addition of purified FabZ 

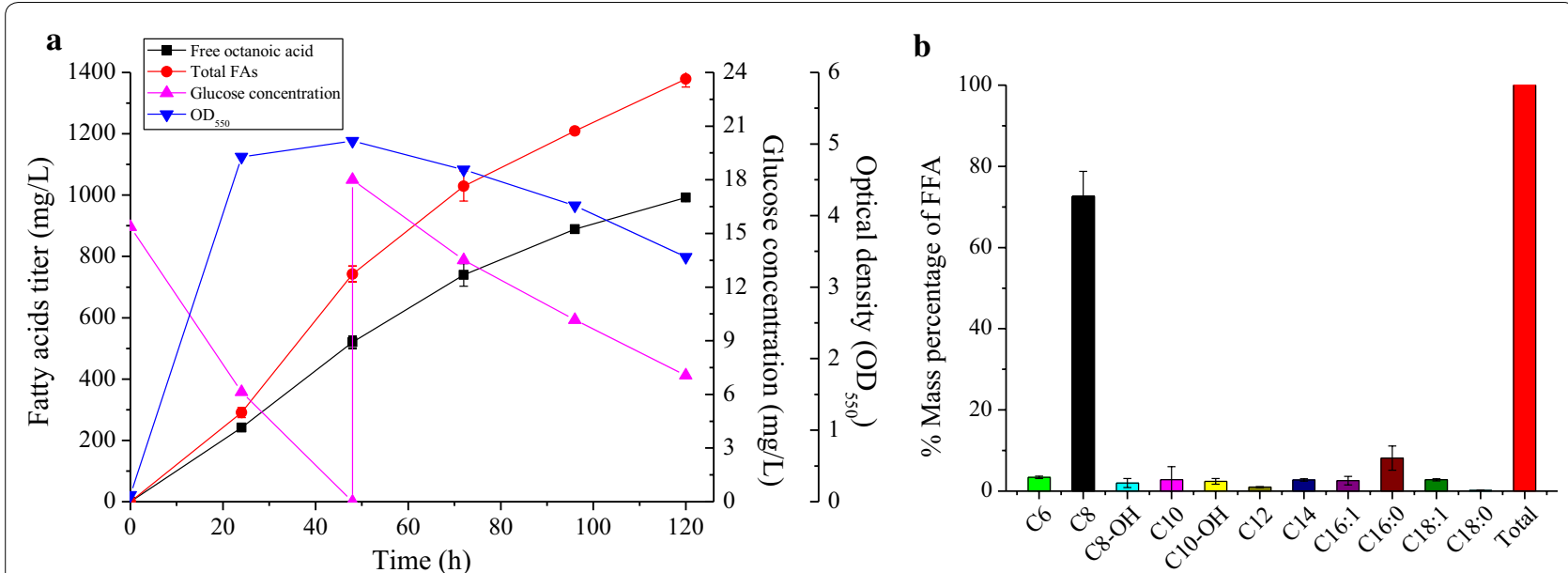

Fig. 5 Fed-batch culture of strain ZEFA (TE10) for free octanoic acid production. a Profile of fed-batch culture. b FFAs distribution in ZEFA (TE10) at the end of culture. Culture was initially performed in $300 \mathrm{~mL} \mathrm{M9+1.5 \% (wt/v)} \mathrm{dextrose} \mathrm{in} \mathrm{500-mL} \mathrm{bioreactor.} \mathrm{An} \mathrm{additional} 9 \mathrm{~mL}$ of fresh $50 \%(\mathrm{wt} / \mathrm{v})$ glucose was added after $48 \mathrm{~h}$, as indicated. Cultures were grown at $30^{\circ} \mathrm{C}$, and the $\mathrm{pH}$ was maintained at 7.0 using $2 \mathrm{M}$ potassium hydroxide (KOH). Air flow rate was controlled at $0.3 \mathrm{~L} / \mathrm{min}$ with $300 \mathrm{rpm}$ as the initial stirring speed. The dissolved oxygen (DO) level was set over $40 \%$ and controlled by changing the stirring speed with the maximum of $600 \mathrm{rpm}$. Octanoic acid titers are the average of at least three replicates with error bars indicating one standard deviation. ZEFA, +fadZ $\triangle$ fadE $\triangle$ fumAC $\triangle a c k A$

enzyme exhibits the highest increase in the rate of FAS reaction among different FAS subunits [50], which agrees with the effectiveness of $+f a b Z$ and the utility of the OptForce prediction. Although $+f a b G$ has been shown effective in production of MCFA [51], it exhibits limited effect on $\mathrm{C} 8$ production in this study, which suggests the differences underlying MCFA and $C 8$ biosynthesis.

In contrast to $+f a b Z,+f a b B$ and $+f a b F$ were found to decrease $\mathrm{C} 8$ production and total fatty acids to some extent. Both $+f a b B$ and $+f a b F$ have been reported to speed up the acyl-ACP elongation, resulting in depletion of C8-ACP levels for C8 production; furthermore, the longer acyl-ACPs feedback inhibits the initiation step of fatty acid biosynthesis [17]. In an attempt to overcome this feedback inhibition by overexpressing $f a b Z$ together with $f a b G$ and $f a b B / f a b F,+f a b G$ and $+f a b B / F$ strains based on $+f a b Z$ were constructed and analyzed for fatty acid production $(+f a b Z G B /+f a b Z G F$, Additional file 2: Figure S1). The overexpression of $f a b Z G B(+f a b Z G B)$ increased $C 8$ and total fatty acid over the wild type but $+f a b Z G F$ did not. Importantly, compared with $+f a b Z$, both $+f a b Z G B$ and $+f a b Z G F$ decreased rather than increased $C 8$ production and total fatty acid production (Table 2). Prior study of dynamic manipulation of FabB and FabF did result in enhanced production of $\mathrm{C} 8$ over the wild type in minimal media [17] but FabZ was not considered in that study. The complex regulation in this recursive biosynthetic system presents a challenge for optimizing $\mathrm{C} 8$ relative to total fatty acid production at the higher titers needed for industrial production.
Individual intervention of genes in Fad module, FUM module, and Ace module shows no significant positive or even negative effects on C8 production (Table 2). It is consistent with the prioritizing orders of OptForce predictions: all of these interventions should be seen effectiveness for $\mathrm{C} 8$ production when in conjunction with the Fab module. $\triangle$ fumAC alone was found to moderately compromise cellular growth and $\mathrm{C} 8$ production (Table 2). One possibility is that $\triangle$ fumAC alone will cause excessive accumulation of acetyl-CoA for compromised TCA cycle which is the main sink for acetyl-CoA catabolic metabolism. In this study, we observed that $+f a b Z / \triangle f a d E$ manipulations based on $\triangle f u m A C$ instead significantly increased cellular growth and $C 8$ production (Additional file 3: Figure S2). This phenomenon can be explained well by $+f a b Z$ which effectively channels excessive acetyl-CoA into $\mathrm{C} 8$ biosynthesis and $\triangle f a d E$ also alleviates further accumulation of acetyl-CoA sourcing from fatty acid degradation. In addition, since the TCA cycle serves as a primary source for ATP and NAD $(\mathrm{P}) \mathrm{H}$ factors, compromised TCA cycle activity from $\triangle$ fum $A C$ might impair cell energy metabolism [52]. Prior studies found that increasing ATP energy supply improved fatty acid production under high cell density condition [53]. Hence, balancing the flux between entering into cell energy metabolism and fatty acid biosynthesis should be cautiously considered.

After combinatorial utilization of Fad, FUM, and Ace modules based on Fab module according to the suggested prioritizing order, we finally obtained the best 
C8 production strain E. coli ZEFA (TE10). Overall, the manipulations of $+f a b Z, \triangle f a d E, \triangle f u m A C$, and $\triangle a c k A$ in ZEFA (TE10) have a synergistic role in increasing C8 production: $+f a b Z$ primarily strengthens $\mathrm{C} 8$ biosynthesis pathway efficiency, $\triangle f a d E$ eliminates $\beta$-oxidation of $\mathrm{C} 8$ degradation, and $\triangle$ fum $A C$ in the TCA cycle and $\triangle a c k A$ in acetate formation maintain a higher acetyl-CoA pool for channeling into $\mathrm{C} 8$ biosynthesis pathway strengthened by $+f a b Z$. All of these interventions enabled the ZEFA (TE10) strain to produce $1 \mathrm{~g} / \mathrm{L}$ of free $\mathrm{C} 8$ in M9 medium, which is the highest titer under minimal medium using E. coli native fatty acid biosynthesis mechanism to our knowledge (Table 1).

It is to be noted that OptForce does not suggest the increased production of acetyl-CoA carboxylase (ACC) for $\mathrm{C} 8$ production, which is supposed to drive more acetyl-CoA flux to malonyl-CoA for C8 biosynthesis. In order to investigate the effect of increasing production of $\mathrm{ACC}$ on $\mathrm{C} 8$ production, $+a c c A B C D$ was performed in our engineered strain. However, no improvement in free C8 production was observed (Additional file 4: Figure S3), indicating that acetyl-CoA carboxylation might be not one of the bottlenecks for $\mathrm{C} 8$ production. This result is consistent with previous observations that increased production of ACC did not lead to significant increase in production of MCFA $[8,16]$.

In this study, besides genetic interventions, we also optimized the culture conditions for $\mathrm{C} 8$ production, e.g., IPTG dosage, $\mathrm{C} / \mathrm{N}$ ratio, and control of $\mathrm{pH}$ at neutral range, all of which were found to contribute to free C8 production. However, although C8 titer and yield in ZEFA (TE10) strain after four rounds of engineering increased significantly compared with the starting strain, it is still lower than the predicted result of OptForce. Specifically, OptForce predicted to yield almost $90 \%$ of the theoretical maximum of $\mathrm{C} 8$ production $(\sim 0.34 \mathrm{~g} / \mathrm{g}$ glucose), while only $11 \%(0.0385 \mathrm{~g} / \mathrm{g}$ glucose $)$ was experimentally achieved in ZEFA (TE10). OptForce predictions are solely based on stoichiometric limitations with no underlying kinetic and regulatory constraints, while these factors are significant contributors towards limiting carbon flux [31,33]. In addition, low catalytic capacity of thioesterase TE10 used here for hydrolysis of octanoyl-ACP, the complex regulation of the recursive fatty acid biosynthesis pathway which complicates the balance of $\mathrm{C} 8$ and longer chain fatty acids at high titers, and importantly, toxicity from the end-product of $\mathrm{C} 8$ might also compromise strain performance and thus C8 titer and yield [49, 54]. Prior research has shown that octanoic acid toxicity increases at lower $\mathrm{pH}$ values, particularly as the media $\mathrm{pH}$ nears the molecule pKa $[49,54]$. Considering that membrane damage is deemed as a fundamental mechanism of fatty acid toxicity, and membrane engineering has proven its effectiveness in alleviating membrane damage from fatty acids [30,55-58], it is reasonable to expect that application of these membrane engineering strategies combined with the metabolic interventions here will contribute to further $\mathrm{C} 8$ improvement in the future.

Recent research about biosynthesis of SCFA (C6-C10, they defined as MCFA there) in E. coli via introduction of the non-native reverse beta-oxidation cycle ( $\mathrm{r}$ - $\mathrm{BOX})$ has led to titers of $>1 \mathrm{~g} / \mathrm{L}$ in rich medium [59]. Heterologous thiolases and trans-enoyl-CoA reductase in conjunction with endogenous $\mathrm{FadB}$ and thioesterase were activated for operation of r-BOX. Although a high titer of SCFA in rich medium was achieved $(3.8 \mathrm{~g} / \mathrm{L})$, the SCFA was a mixture of $\mathrm{C} 4, \mathrm{C} 6, \mathrm{C} 8$, and $\mathrm{C} 10$. Due to the r-BOX inherent characteristic of operation in an iterative manner [14, $60,61]$, it may be challenging to narrow down the MCFA to a specific length. In this study, we selectively produced C8 from the other fatty acids, which then enables more efficient recovery of the desired product.

In summary, by implementing a minimal set of metabolic interventions, we have successfully engineered $E$. coli MG1655 inherent metabolism network and achieved the high production and selectivity of $\mathrm{C} 8$. Besides $\mathrm{C} 8$, this strategy which integrates computational strain design and experimental study is expected to be applicable to production of other biorenewables.

\section{Conclusions}

In this study, a combination of integrated computational and experimental approach was performed to manipulate the E. coli native metabolic network for octanoic acid (C8) production. Four interventions were subsequently predicted by a customized OptForce methodology. Then, all the ten associated candidate proteins were regulated individually and combinatorially. +FabZ was identified as the most prominent individual intervention and the final combinatorial strain based on +FabZ eventually produced $1 \mathrm{~g} / \mathrm{L}$ of $\mathrm{C} 8$ with high selectivity in minimum medium using glucose as single carbon. This work underlines the significance of integration of computational strain design and experimental testing in rewiring microbial metabolism for octanoic acid production. This result besides other studies using OptForce in strain design demonstrates that this strategy may be also applicable to engineering $E$. coli for production of other customized biorenewable chemicals or biofuels.

\section{Methods}

Strains and plasmids

All plasmids and strains used in this study are listed in Table 4. Primers can be found in Additional file 5: Table S2. E. coli K-12 MG1655 was used as the base strain 
Table 4 Strains and plasmids used in this study

\begin{tabular}{|c|c|c|}
\hline Strains/plasmids & Genetic characteristics & Source \\
\hline \multicolumn{3}{|l|}{ Strains } \\
\hline MG1655 & Wild-type E. coli K1-12 strain & Lab collection \\
\hline$+f a d B$ & MG1655, mgsA::M1-93-fadB & This study \\
\hline+ fadF & MG1655, mgsA::M1-93-fadF & This study \\
\hline$+f a d G$ & MG1655, mgsA::M1-93-fadG & This study \\
\hline+ fad $Z$ & MG1655, mgsA::M1-93-fadZ & This study \\
\hline$+f a d Z G B$ & MG1655, mgsA::M1-93-fadZ-RBS1-fabG-RBS2-fabB & This study \\
\hline+ fadZGF & MG1655, mgsA::M1-93-fadZ-RBS1-fabG-RBS2-fabF & This study \\
\hline$\triangle f a d E$ & MG1655, $\triangle$ fadE & This study \\
\hline$\triangle f a d A$ & MG1655, $\triangle$ fadA & This study \\
\hline$\triangle f a d B$ & MG1655, $\triangle \mathrm{fadB}$ & This study \\
\hline$\triangle f u m A C$ & MG1655, $\triangle$ fumAC & This study \\
\hline$\triangle a c k A$ & MG1655, $\triangle a c k A$ & This study \\
\hline$\Delta p t a$ & MG1655, $\Delta p t a$ & This study \\
\hline$+f a d Z \triangle f a d E$ & MG1655, mgsA::M1-93-fadZ, $\triangle$ fadE & This study \\
\hline$+f a d Z \triangle f a d A$ & MG1655, mgsA::M1-93-fadZ, $\triangle$ fadA & This study \\
\hline$+f a d Z \triangle f a d B$ & MG1655, mgsA::M1-93-fadZ, $\triangle$ fadB & This study \\
\hline+ fadZ $\triangle$ fadE $\triangle$ fumAC & MG1655, mgsA::M1-93-fadZ, $\triangle$ fadE, $\triangle$ fumAC & This study \\
\hline+ fadZ $\triangle$ fadE $\triangle$ fumAC $\triangle a c k A(Z E F A)$ & MG1655, mgsA::M1-93-fadZ, $\triangle$ fadE, $\triangle$ fumAC, $\triangle a c k A$ & This study \\
\hline+ fadZ $\triangle$ fadE $\triangle$ fumAC $\triangle p t a$ & MG1655, mgsA::M1-93-fadZ, $\triangle f a d E, \triangle f u m A C, \triangle p t a$ & This study \\
\hline \multicolumn{3}{|l|}{ Plasmids } \\
\hline pJMYEEI82564 (TE10) & pTrc-EEl82564 thioesterase from Anaerococcus tetradius, Amp ${ }^{r}$ & [49] \\
\hline
\end{tabular}

in this study. The scarless CRISPR-Cas9 approach was employed to perform genetic deletion and tuning gene expression [34]. For increasing expression of $f a b$ genes $(f a b B, f a b F, f a b G, f a b Z)$, a second copy of each gene with a strong constitutive M1-93 promoter [30,58] was inserted into chromosomal DNA of MG1655 at $m g s A$ site. For octanoic acid production, the pJMYEEI82564 plasmid [30, 62], harboring the Anaerococcus tetradius thioesterase (TE10) that primarily hydrolyzes octanoylACP [29], was transformed into our engineered strains. When necessary, ampicillin, kanamycin, and chloramphenicol were used at a final concentration of 100, 50, and $34 \mathrm{mg} / \mathrm{L}$, respectively.

\section{Using OptForce for octanoic acid overproduction}

OptForce optimization algorithm was used for identifying a prioritized intervention strategy for octanoate overproduction. The $i$ AF1260 genome-scale metabolic (GSM) model in E. coli [63] was used for all the simulations under aerobic minimal conditions with glucose as the sole carbon substrate. A couple of modifications were made to the original GSM model (for example, activation of the beta-oxidation pathway reactions regulated off in the original model) based on prior experimental observations [13, 31]. Details of the modification, along with the flux bounds for exchange reactions for media metabolites, have been tabulated in Additional file 6: Text S1. Description of the sequential steps for implementation of the entire OptForce algorithm, along with details of the optimization formulations in each step, has been described elsewhere $[31,64]$. In short, ${ }^{13} \mathrm{C}$ MFA for a wild-type E. coli strain [31] is imposed on the GSM model to characterize its native phenotype. This phenotype is contrasted to an overproduction phenotype producing octanoate at least $90 \%$ of its theoretical maximum capability (as well as a minimum growth-rate of $10 \%$ of its theoretical maximum). The contrast reveals a subset of reactions that "MUST" be altered (directly or indirectly) from their native activity to ensure octanoate overproduction. Note that the MUST set of reactions can be identified for increasing levels of complexity. At the "singles" level, individual reactions whose overproduction flux range has no overlap with the corresponding flux range in the native phenotype (either as an up-regulation or a down-regulation) are identified (i.e., MUST singles). The same analysis can be extended to identify pairs, triplets, or higher combination of reactions whose sum (or difference) of fluxes in the overproduction phenotype completely departs from the corresponding flux combination in the wild-type phenotype, even though their individual flux ranges have an overlap. This leads towards identification of MUST pairs, triples, and higher 
order sets. For the current simulation, the analysis as limited to doubles due to relative abundance of target reactions identified in the MUST sets. Finally, a bilevel optimization algorithm identifies a prioritized list of interventions from the MUST set of reactions that must be directly engineered to improve octanoate yield. See Additional file 6: Text S1 for expanded execution guidelines. The SBML file for iAF1260 model was obtained from the BIGG models repository (http://bigg.ucsd.edu/ models/iAF1260). In addition, GAMS compatible files for the iAF1260 model and the OptForce code in GAMS for current simulation have been included in Additional file 7 . Further description of the protocol can be found from http://maranasgroup.com/software.htm.

\section{In vivo production of $\mathrm{C} 8$ in shake flask}

Individual colonies were selected from Luria Broth (LB) plates with ampicillin, inoculated into $3 \mathrm{~mL}$ of $\mathrm{LB}$ liquid medium with ampicillin for $4 \mathrm{~h}$. Then, $0.5 \mathrm{~mL}$ of culture was added to $10 \mathrm{~mL} \mathrm{M9}\left(0.8 \mathrm{~g} / \mathrm{L} \mathrm{NH}_{4} \mathrm{Cl}, 0.5 \mathrm{~g} / \mathrm{L}\right.$ $\mathrm{NaCl}, 7.52 \mathrm{~g} / \mathrm{L} \quad \mathrm{Na}_{2} \mathrm{HPO}_{4}, 3.0 \mathrm{~g} / \mathrm{L} \quad \mathrm{KH}_{2} \mathrm{PO}_{4}, 0.24 \mathrm{~g} / \mathrm{L}$ $\mathrm{MgSO}_{4}, 11.1 \mathrm{mg} / \mathrm{L} \mathrm{CaCl}, 1 \mathrm{mg} / \mathrm{L}$ thiamine $\mathrm{HCl}$, and trace elements containing $166.7 \mu \mathrm{g} / \mathrm{L} \quad \mathrm{FeCl}_{3} \cdot 6 \mathrm{H}_{2} \mathrm{O}$, $1.8 \mu \mathrm{g} / \mathrm{L} \mathrm{ZnSO}_{4} \cdot 7 \mathrm{H}_{2} \mathrm{O}, 1.2 \mu \mathrm{g} / \mathrm{L} \mathrm{CuCl} \mathrm{Cu}_{2} \cdot 2 \mathrm{H}_{2} \mathrm{O}, 1.2 \mu \mathrm{g} / \mathrm{L}$ $\mathrm{MnSO}_{4} \cdot 2 \mathrm{H}_{2} \mathrm{O}, 1.8 \mu \mathrm{g} / \mathrm{L} \mathrm{CoCl}_{2} \cdot 6 \mathrm{H}_{2} \mathrm{O}$, and $0.223 \mathrm{mg} / \mathrm{L}$ $\mathrm{Na}_{2}$ EDTA $\cdot 2 \mathrm{H}_{2} \mathrm{O}$ ) with $1.5 \%$ (wt/v) dextrose at $30{ }^{\circ} \mathrm{C}$, $220 \mathrm{rpm}$ overnight for seed-culture preparation. Midlog phase seed-culture was transferred into $40 \mathrm{~mL} \mathrm{M9}$ with $1.5 \%(\mathrm{wt} / \mathrm{v})$ dextrose medium at the final concentration of $\mathrm{OD}_{550} \sim 0.1$. For inducing TE10 gene expression, isopropyl- $\beta$-D-thiogalactopyranoside (IPTG) was typically added at a final concentration of $1 \mathrm{mM}$ when the $\mathrm{OD}_{550}$ reached $0.4-0.5$. Cultures were grown in 250$\mathrm{mL}$ baffled flasks with initial pH 7.0 at $30{ }^{\circ} \mathrm{C}, 220 \mathrm{rpm}$ for $72 \mathrm{~h}$. In M9 minimal medium, $0.8 \mathrm{~g} / \mathrm{L}$ of ammonium chloride $\left(\mathrm{NH}_{4} \mathrm{Cl}\right)$ was used as the only nitrogen source, resulting in a standard $\mathrm{C} / \mathrm{N}$ ratio of 18.8 . For changing the $\mathrm{C} / \mathrm{N}$ ratio, the glucose (carbon source) concentration was fixed at $15 \mathrm{~g} / \mathrm{L}$ and the $\mathrm{NH}_{4} \mathrm{Cl}$ (carbon source) was added at final concentrations of $0.2,0.4,1.6$, and $2.4 \mathrm{~g} / \mathrm{L}$, corresponding to $\mathrm{C} / \mathrm{N}$ ratios of $75,37.5,9.4$, and 6.25 , respectively.

\section{In vivo production of $\mathrm{C} 8$ in bioreactor}

Batch cultures were performed in $300 \mathrm{~mL} \mathrm{M} 9+1.5 \%$ (wt/v) dextrose in 500-mL bioreactor (INFORS HT). Cultures were grown at $30{ }^{\circ} \mathrm{C}$, and the $\mathrm{pH}$ was maintained at 7.0 by adding $2.0 \mathrm{M}$ potassium hydroxide $(\mathrm{KOH})$. Air flow rate was initially maintained at $0.3 \mathrm{~L} / \mathrm{min}$. The dissolved oxygen (DO) level was set over $40 \%$ and controlled by changing the stirring speed, with a maximum value of $600 \mathrm{rpm}$. Similar operations were performed for fed-batch culture, the only difference being that a $9 \mathrm{~mL}$ of $50 \%(w t / v)$ glucose was fed to the cultures after $48-\mathrm{h}$ cultivation.

\section{Determination of carboxylic acid titers}

Carboxylic acid production was quantified by an Agilent 7890 gas chromatograph equipped with an Agilent 5975 mass spectroscope using mass spectrometer (GC-MS) after carboxylic acid extraction [17]. Briefly, $1 \mathrm{~mL}$ of culture was mixed with $125 \mu \mathrm{L}$ of $10 \%$ (wt/v) sodium chloride and $125 \mu \mathrm{L}$ of acetic acid. Then, $20 \mu \mathrm{L}$ of $1 \mathrm{mg} / \mathrm{mL}$ internal standards (C9:0/C13:0/C15:0/C17:0) was added into the mixture followed by $500 \mu \mathrm{L}$ of ethyl acetate. The mixture was vortexed for $30 \mathrm{~s}$ and then centrifuged at $16,000 \times g$ for $10 \mathrm{~min}$. After that, $250 \mu \mathrm{L}$ of top organic phase was transferred to a new glass tube followed by addition of $2.25 \mathrm{~mL}$ of ethanol:hydrochloric acid (30:1 $\mathrm{v} / \mathrm{v})$. After incubation at $55^{\circ} \mathrm{C}$ for $1 \mathrm{~h}, 1.25 \mathrm{~mL}$ of double-distilled water $\left(\mathrm{ddH}_{2} \mathrm{O}\right)$ and $1.25 \mathrm{~mL}$ of hexane were added, vortexed, and centrifuged at $2000 \times g$ for $2 \mathrm{~min}$. The top hexane layer was then transferred and analyzed by GC-MS. The temperature for GC-MS analysis was initially held at $50{ }^{\circ} \mathrm{C}$ for $2 \mathrm{~min}$, ramped to $200{ }^{\circ} \mathrm{C}$ at $25{ }^{\circ} \mathrm{C} / \mathrm{min}$, held for $1 \mathrm{~min}$, and then raised to $315^{\circ} \mathrm{C}$ at $25{ }^{\circ} \mathrm{C} / \mathrm{min}$, held for $2 \mathrm{~min}$. Helium was used as a carrier gas and the flow rate was set as $1 \mathrm{~mL} / \mathrm{min}$ through a DB-5MS separation column (30 m, $0.25 \mathrm{~mm}$ ID, $0.25 \mu \mathrm{m}$, Agilent).

\section{Statistical analysis}

The two-tailed $t$ test method (two-sample equal variance, homoscedastic) was employed to analyze the statistical significance of all the data in this study and $P$ value $<0.05$ is deemed statistically significant.

\section{Additional files}

Additional file 1: Table S1. The simulations predicted by the iAF1260 genome-scale metabolic model of E. coli.

Additional file 2: Figure S1. Schematic of overexpression of $f a b Z, f a b G$, $\mathrm{fabB/F}$ together for $\mathrm{C} 8$ production.

Additional file 3: Figure S2. Comparison of $\triangle$ fumAC alone and $+f a b Z$ $\triangle$ fadE $\triangle$ fumAC combination in cellular growth during C8 production.

Additional file 4: Figure S3. Effects of overexpression of acc $A B C D$ on $C 8$ production.

Additional file 5: Table S2. Primers and sequence used in this study. Additional file 6: Text S1. Details of OptForce simulation for overproduction of octanoate in E. coli.

Additional file 7. GAMS compatible files for the iAF1260 model and the OptForce code.

\section{Abbreviations}

SCFA: short-chain fatty acid; MCFA: medium-chain fatty acid; C8: octanoic acid; IPTG: isopropyl $\beta$-D-1-thiogalactopyranoside; ZEFA: +fadZ $\triangle$ fadE $\triangle$ fumAC $\triangle a c k A ; T E 10$ : thioesterase from Anaerococcus tetradius. 


\section{Authors' contributions}

JVS and CDM designed research; ZT, JMY, and KB performed research; AC ran the OptForce procedure; ZT, JMY, JVS, CDM, LRJ, and JVS analyzed data and wrote the paper. All authors reviewed this manuscript. All authors read and approved the final manuscript.

\section{Author details}

1 Department of Chemical and Biological Engineering, lowa State University, 3031 Sweeney, Ames, IA 50011, USA. ${ }^{2}$ Department of Chemical Engineering, The Pennsylvania State University, University Park, PA 16802, USA

\section{Acknowledgements}

We thank Addgene and Dr. Sheng Yang for providing pCas and pTargetF plasmids for performing the CRISPR-Cas9 technique. We thank ISU W.M. Keck Metabolomics Research Laboratory for help with GC-MS analysis.

\section{Competing interests}

The authors declare that they have no competing interests.

\section{Availability of data and materials}

The dataset supporting the conclusions of this article is included within the article and its additional files.

\section{Consent for publication}

Not applicable.

\section{Ethics approval and consent to participate}

Not applicable.

\section{Funding}

This work was supported by the NSF Engineering Research Center for Biorenewable Chemicals (CBiRC), NSF Award number EEC-0813570. The funders had no role in study design, data collection and analysis, decision to publish, or preparation of the manuscript.

\section{Publisher's Note}

Springer Nature remains neutral with regard to jurisdictional claims in published maps and institutional affiliations.

Received: 20 November 2017 Accepted: 13 March 2018 Published online: 02 April 2018

\section{References}

1. Larson ED. A review of life-cycle analysis studies on liquid biofuel systems for the transport sector. Energy Sustain Dev. 2006;10:109-26.

2. Gallezot P. Process options for converting renewable feedstocks to bioproducts. Green Chem. 2007;9:295-302.

3. Wymann MP, Schneiter R. Lipid signalling in disease. Nat Rev Mol Cell Biol. 2008:9:162-76.

4. Desbois AP, Smith VJ. Antibacterial free fatty acids: activities, mechanisms of action and biotechnological potential. Appl Microbiol Biotechnol. 2010;85:1629-42

5. Black BA, Zannini E, Curtis JM, Ganzle MG. Antifungal hydroxy fatty acids produced during sourdough fermentation: microbial and enzymatic pathways, and antifungal activity in bread. Appl Environ Microbiol. 2013;79:1866-73

6. Sokolsky-Papkov M, Shikanov A, Ezra A, Vaisman B, Domb AJ. Fatty acidbased biodegradable polymers: synthesis and applications. Polyme Degrad Perform. 2009;1004:60-9.

7. Lopez-Ruiz JA, Davis RJ. Decarbonylation of heptanoic acid over carbonsupported platinum nanoparticles. Green Chem. 2014;16:683-94.

8. Lennen RM, Braden DJ, West RM, Dumesic JA, Pfleger BF. A process for microbial hydrocarbon synthesis: overproduction of fatty acids in Escherichia coli and catalytic conversion to alkanes. Biotechnol Bioeng 2010;106:193-202.

9. Korstanje TJ, van der Vlugt II, Elsevier CJ, de Bruin B. Hydrogenation of carboxylic acids with a homogeneous cobalt catalyst. Science. 2015:350:298-302.
10. Maki-Arvela P, Kubickova I, Snare M, Eranen K, Murzin DY. Catalytic deoxygenation of fatty acids and their derivatives. Energy Fuels. 2007;21:30-41.

11. Chatterjee A, Jensen VR. A heterogeneous catalyst for the transformation of fatty acids to a-olefins. ACS Catal. 2017;7:2543-7.

12. Tee TW, Chowdhury A, Maranas CD, Shanks JV. Systems metabolic engineering design: fatty acid production as an emerging case study. Biotechnol Bioeng. 2014:111:849-57.

13. Steen EJ, Kang YS, Bokinsky G, Hu ZH, Schirmer A, McClure A, del Cardayre SB, Keasling JD. Microbial production of fatty-acid-derived fuels and chemicals from plant biomass. Nature. 2010;463:559-62.

14. Clomburg JM, Vick JE, Blankschien MD, Rodriguez-Moya M, Gonzalez R. A synthetic biology approach to engineer a functional reversal of the betaoxidation cycle. ACS Synth Biol. 2012;1:541-54.

15. Liu H, Yu C, Feng DX, Cheng T, Meng X, Liu W, Zou HB, Xian M. Production of extracellular fatty acid using engineered Escherichia coli. Microb Cell Fact. 2012;11:41.

16. Lu XF, Vora H, Khosla C. Overproduction of free fatty acids in E. coli: implications for biodiesel production. Metab Eng. 2008;10:333-9.

17. Torella JP, Ford TJ, Kim SN, Chen AM, Way JC, Silver PA. Tailored fatty acid synthesis via dynamic control of fatty acid elongation. Proc Natl Acad Sci USA. 2013;110:11290-5.

18. Xu P, Gu Q, Wang W, Wong L, Bower AG, Collins CH, Koffas MA. Modular optimization of multi-gene pathways for fatty acids production in E. coli. Nat Commun. 2013;4:1409.

19. Zheng Y, Li L, Liu Q, Qin W, Yang J, Cao Y, Jiang X, Zhao G, Xian M. Boosting the free fatty acid synthesis of Escherichia coli by expression of a cytosolic Acinetobacter baylyi thioesterase. Biotechnol Biofuels. 2012;5:76.

20. Knothe $\mathrm{G}$. Improving biodiesel fuel properties by modifying fatty ester composition. Energy Environ Sci. 2009:2:759-66.

21. Choi JW, Da Silva NA. Improving polyketide and fatty acid synthesis by engineering of the yeast acetyl-CoA carboxylase. J Biotechnol. 2014;187:56-9.

22. Park J, Rodriguez-Moya M, Li M, Pichersky E, San KY, Gonzalez R. Synthesis of methyl ketones by metabolically engineered Escherichia coli. J Ind Microbiol Biotechnol. 2012;39:1703-12.

23. Wu H, Karanjikar M, San KY. Metabolic engineering of Escherichia coli for efficient free fatty acid production from glycerol. Metab Eng 2014;25:82-91.

24. Zhu Z, Zhou YJ, Krivoruchko A, Grininger M, Zhao ZK, Nielsen J. Expanding the product portfolio of fungal type I fatty acid synthases. Nat Chem Biol. 2017;13:360-2

25. Gajewski J, Pavlovic R, Fischer M, Boles E, Grininger M. Engineering fungal de novo fatty acid synthesis for short chain fatty acid production. Nat Commun. 2017:8:14650.

26. Van Eerten-Jansen MCAA, Ter Heijne A, Grootscholten TIM, Steinbusch KJJ, Sleutels THJA, Hamelers HVM, Buisman CJN. Bioelectrochemical production of caproate and caprylate from acetate by mixed cultures. ACS Sustain Chem Eng. 2013;1:513-8.

27. Steinbusch KJJ, Hamelers HVM, Plugge CM, Buisman CJN. Biological formation of caproate and caprylate from acetate: fuel and chemical production from low grade biomass. Energy Environ Sci. 2011:4:216-24.

28. Spirito CM, Richter H, Rabaey K, Stams AJ, Angenent LT. Chain elongation in anaerobic reactor microbiomes to recover resources from waste. Curr Opin Biotechnol. 2014;27:115-22.

29. Jing FY, Cantu DC, Tvaruzkova J, Chipman JP, Nikolau BJ, Yandeau-Nelson MD, Reilly PJ. Phylogenetic and experimental characterization of an acyl-ACP thioesterase family reveals significant diversity in enzymatic specificity and activity. BMC Biochem. 2011;12:44.

30. Tan Z, Yoon JM, Nielsen DR, Shanks JV, Jarboe LR. Membrane engineering via trans unsaturated fatty acids production improves Escherichia coli robustness and production of biorenewables. Metab Eng 2016:35:105-13.

31. Ranganathan S, Tee TW, Chowdhury A, Zomorrodi AR, Yoon JM, Fu Y, Shanks JV, Maranas CD. An integrated computational and experimental study for overproducing fatty acids in Escherichia coli. Metab Eng 2012;14:687-704

32. Xu P, Ranganathan S, Fowler ZL, Maranas CD, Koffas MAG. Genomescale metabolic network modeling results in minimal interventions that cooperatively force carbon flux towards malonyl-CoA. Metab Eng. 2011;13:578-87. 
33. Ranganathan S, Suthers PF, Maranas CD. OptForce: an optimization procedure for identifying all genetic manipulations leading to targeted overproductions. PLoS Comput Biol. 2010;6(4):e1000744.

34. Jiang Y, Chen B, Duan CL, Sun BB, Yang JJ, Yang S. Multigene editing in the Escherichia coli genome via the CRISPR-Cas9 system. Appl Environ Microbiol. 2015;81:2506-14.

35. Janssen HJ. Fatty acid synthesis in Escherichia coli and its applications towards the production of fatty acid based biofuels. Biotechnol Biofuels 2014;7(1):7.

36. Wu G, Yan Q, Jones JA, Tang YJ, Fong SS, Koffas MAG. Metabolic burden: cornerstones in synthetic biology and metabolic engineering applications. Trends Biotechnol. 2016:34:652-64.

37. Li Q, Fan F, Gao X, Yang C, Bi C, Tang J, Liu T, Zhang X. Balanced activation of IspG and IspH to eliminate MEP intermediate accumulation and improve isoprenoids production in Escherichia coli. Metab Eng. 2017:44:13-21.

38. Yadav VG, De Mey M, Lim CG, Ajikumar PK, Stephanopoulos G. The future of metabolic engineering and synthetic biology: towards a systematic practice. Metab Eng. 2012;14:233-41.

39. Pitera DJ, Paddon CJ, Newman JD, Keasling JD. Balancing a heterologous mevalonate pathway for improved isoprenoid production in Escherichia coli. Metab Eng. 2007;9:193-207.

40. Tan Z, Chen J, Zhang X. Systematic engineering of pentose phosphate pathway improves Escherichia coli succinate production. Biotechnol Biofuels. 2016;9:262.

41. Ajikumar PK, Xiao WH, Tyo KE, Wang Y, Simeon F, Leonard E, Mucha O, Phon TH, Pfeifer B, Stephanopoulos G. Isoprenoid pathway optimization for Taxol precursor overproduction in Escherichia coli. Science. 2010:330:70-4.

42. Tseng CP, Yu CC, Lin HH, Chang CY, Kuo JT. Oxygen- and growth ratedependent regulation of Escherichia coli fumarase (FumA, FumB, and FumC) activity. J Bacteriol. 2001;183:461-7.

43. Tseng CP. Regulation of fumarase (fumB) gene expression in Escherichia coli in response to oxygen, iron and heme availability: role of the $\operatorname{arcA}$, fur, and hemA gene products. FEMS Microbiol Lett. 1997;157:67-72.

44. Li M, Zhang X, Agrawal A, San KY. Effect of acetate formation pathway and long chain fatty acid CoA-ligase on the free fatty acid production in E. coli expressing acy-ACP thioesterase from Ricinus communis. Metab Eng. 2012;14:380-7.

45. Baneyx F. Recombinant protein expression in Escherichia coli. Curr Opin Biotechnol. 1999;10:411-21.

46. Sriubolmas N, Panbangred W, Sriurairatana S, Meevootisom V. Localization and characterization of inclusion bodies in recombinant Escherichia coli cells overproducing penicillin G acylase. Appl Microbiol Biotechnol. 1997:47:373-8

47. Zhang X, Li M, Agrawal A, San KY. Efficient free fatty acid production in Escherichia coli using plant acyl-ACP thioesterases. Metab Eng. 2011;13:713-22

48. Singhasuwan S, Choorit W, Sirisansaneeyakul S, Kokkaew N, Chisti Y. Carbon-to-nitrogen ratio affects the biomass composition and the fatty acid profile of heterotrophically grown Chlorella sp. TISTR 8990 for biodiesel production. J Biotechnol. 2015;216:169-77.

49. Jarboe LR, Royce LA, Liu P. Understanding biocatalyst inhibition by carboxylic acids. Front Microbiol. 2013;4:272.

50. Yu X, Liu T, Zhu F, Khosla C. In vitro reconstitution and steady-state analysis of the fatty acid synthase from Escherichia coli. Proc Natl Acad Sci USA. 2011;108:18643-8.

51. Lee $S$, Jung Y, Lee S, Lee J. Correlations between FAS elongation cycle genes expression and fatty acid production for improvement of long-chain fatty acids in Escherichia coli. Appl Biochem Biotechnol. 2013;169:1606-19.

52. He L, Xiao Y, Gebreselassie N, Zhang F, Antoniewiez MR, Tang YJ, Peng L. Central metabolic responses to the overproduction of fatty acids in Escherichia coli based on ${ }^{13} \mathrm{C}$-metabolic flux analysis. Biotechnol Bioeng. 2014;111:575-85.

53. Liu D, Wan N, Zhang F, Tang YJ, Wu SG. Enhancing fatty acid production in Escherichia coli by Vitreoscilla hemoglobin overexpression. Biotechnol Bioeng. 2017;114:463-7.

54. Royce LA, Liu P, Stebbins MJ, Hanson BC, Jarboe LR. The damaging effects of short chain fatty acids on Escherichia coli membranes. Appl Microbiol Biotechnol. 2013;97:8317-27.

55. Lennen RM, Pfleger BF. Modulating membrane composition alters free fatty acid tolerance in Escherichia coli. PLoS ONE. 2013;8(1):e54031.

56. San KY, Han S, Li W, Li M, Li Z. Fatty acids with mg transporter and mg. US Patent. 20150259712 A1.

57. Sherkhanov S, Korman TP, Bowie JU. Improving the tolerance of Escherichia coli to medium-chain fatty acid production. Metab Eng. 2014;25:1-7.

58. Tan Z, Black W, Yoon JM, Shanks JV, Jarboe LR. Improving Escherichia coli membrane integrity and fatty acid production by expression tuning of FadL and OmpF. Microb Cell Fact. 2017;16:38

59. Wu J, Zhang $X, X i a X$, Dong $M$. A systematic optimization of medium chain fatty acid biosynthesis via the reverse beta-oxidation cycle in Escherichia coli. Metab Eng. 2017:41:115-24.

60. Dellomonaco C, Clomburg JM, Miller EN, Gonzalez R. Engineered reversal of the beta-oxidation cycle for the synthesis of fuels and chemicals. Nature. 2011;476:355-9.

61. Kim S, Clomburg JM, Gonzalez R. Synthesis of medium-chain length (C6C10) fuels and chemicals via beta-oxidation reversal in Escherichia coli. J Ind Microbiol Biotechnol. 2015;42:465-75.

62. Royce LA, Yoon JM, Chen Y, Rickenbach E, Shanks JV, Jarboe LR. Evolution for exogenous octanoic acid tolerance improves carboxylic acid production and membrane integrity. Metab Eng. 2015:29:180-8.

63. Feist AM, Henry CS, Reed JL, Krummenacker M, Joyce AR, Karp PD, Broadbelt LJ, Hatzimanikatis V, Palsson BO. A genome-scale metabolic reconstruction for Escherichia coli K-12 MG1655 that accounts for 1260 ORFs and thermodynamic information. Mol Syst Biol. 2007;3:121.

64. Chowdhury A, Zomorrodi AR, Maranas CD. Bilevel optimization techniques in computational strain design. Comput Chem Eng. 2015:72:363-72.

\section{Submit your next manuscript to BioMed Central and we will help you at every step:}

- We accept pre-submission inquiries

- Our selector tool helps you to find the most relevant journal

- We provide round the clock customer support

- Convenient online submission

- Thorough peer review

- Inclusion in PubMed and all major indexing services

- Maximum visibility for your research

Submit your manuscript at www.biomedcentral.com/submit
C BioMed Central 